Supporting Information

\title{
Highly Strained Au-Ag-Pd Alloy Nanowires for Boosted Electrooxidation of Biomass-Derived Alcohols
}

\footnotetext{
Shumeng Zhang, ${ }^{\dagger}$ Kai Liu, ${ }^{\dagger}$ Zhaojun Liu, ${ }^{\dagger}$ Moxuan Liu, ${ }^{\dagger}$ Zhixue Zhang, ${ }^{\dagger}$ Zhun Qiao, ${ }^{\dagger}$ Liang Ming, ${ }^{\ddagger}$ and Chuanbo Gao ${ }^{\dagger *}$

Center for Materials Chemistry, Frontier Institute of Science and Technology, and State Key Laboratory of Multiphase Flow in Power Engineering, Xi'an Jiaotong University, Xi'an, Shaanxi 710054, China.

*Fengcheng Advanced Energy Materials Research Institute, Ningbo, Zhejiang 315500, China *Email: gaochuanbo@,mail.xjtu.edu.cn (C.G.)
} 


\section{Experimental Section}

Chemicals. Silver nitrate $\left(\mathrm{AgNO}_{3}, 99 \mathrm{wt} \%\right)$, chloroauric acid $\left(\mathrm{HAuCl}_{4}\right)$, sodium chloride $(\mathrm{NaCl}), \mathrm{N}, \mathrm{N}-$ dimethylformamide (DMF), polyvinylpyrrolidone (PVP, Mw 40000) were purchased from Sigma-Aldrich. Palladium acetylacetonate $\operatorname{Pd}(\mathrm{acac})_{2}$ (acac: acetylacetone) was purchased from Innochem. $N, N$-diethylhydroxylamine (DEHA) was purchased from Adamas-beta. Ethanol, ethylene glycol (EG), and glycerol was purchased from Aladdin. Potassium hydroxide (KOH, 85wt \%) was purchased from Acros. All chemicals were used as purchased without further purification.

Synthesis of highly strained ultrathin Au-Ag-Pd alloy nanowires. In a typical synthesis of Au-AgPd alloy nanowires (Pd-hs-37.2\%), $600 \mu \mathrm{L}$ of PVP ( $5 \mathrm{wt} \%$ in DMF), $90 \mu \mathrm{L}$ of $\mathrm{AgNO}_{3}\left(0.1 \mathrm{M}^{\text {in } \mathrm{H}_{2} \mathrm{O} \text { ), } 36}\right.$ $\mu \mathrm{L}$ of $\mathrm{HAuCl}_{4}\left(0.25 \mathrm{M}\right.$ in DMF), $240 \mu \mathrm{L}$ of DEHA and $360 \mu \mathrm{L}$ of $\mathrm{Pd}(\mathrm{acac})_{2}(0.033 \mathrm{M}$ in DMF) were added to $25 \mathrm{~mL}$ of DMF in sequence in a flask in an ice-water bath $\left(0^{\circ} \mathrm{C}\right)$ and stirred for $5 \mathrm{~min}$. The reaction solution was then transferred to a $50{ }^{\circ} \mathrm{C}$ water bath and stirred for another $16 \mathrm{~h}$. The resulting ultrathin Au-Ag-Pd alloy nanowires were collected by centrifugation and washed with $\mathrm{H}_{2} \mathrm{O}$. The molar fraction of $\mathrm{Pd}$ in the nanowires was tuned by adjusting the concentration of $\mathrm{Pd}(\mathrm{acac})_{2}$ in the precursor.

\section{Synthesis of less strained ultrathin Au-Ag-Pd alloy nanowires.}

The less strain Au-Ag-Pd alloy nanowires were synthesized by growing Pd on Au-Ag alloy nanowires with average diameters of 1.8 and $2.9 \mathrm{~nm}$. The Au-Ag alloy nanowires (thickness, $\sim 1.8 \mathrm{~nm}$ ) were first synthesized according to our previously reported protocol. ${ }^{1}$ Typically, $3 \mathrm{~mL}$ of PVP ( $5 \mathrm{wt} \%$ in DMF), 450 $\mu \mathrm{L}$ of $\mathrm{AgNO}_{3}(0.1 \mathrm{M}), 450 \mu \mathrm{L}$ of $\mathrm{NaCl}(1 \mathrm{M}), 1.2 \mathrm{~mL}$ of DEHA, $180 \mu \mathrm{L}$ of $\mathrm{HAuCl}_{4}(0.25 \mathrm{M}$ in DMF) were added to $63 \mathrm{~mL}$ of DMF in sequence in an ice-water bath $\left(0^{\circ} \mathrm{C}\right)$ and stirred for $5 \mathrm{~min}$. The reaction solution was then transferred into a $50{ }^{\circ} \mathrm{C}$ water bath and stirred for another $1 \mathrm{~h}$. The resulting ultrathin Au-Ag alloy nanowires were redispersed in $25 \mathrm{~mL}$ of DMF ("seeds" for the subsequent growth of Pd). In a typical synthesis of Pd- $l s-28.1 \%$ nanowires, $240 \mu \mathrm{L}$ of PVP ( $5 \mathrm{wt} \%$ in DMF), $5 \mathrm{~mL}$ of the $\mathrm{Au}-\mathrm{Ag}$ nanowires in DMF, $107 \mu \mathrm{L}$ of $\mathrm{Pd}(\mathrm{acac})_{2}(0.033 \mathrm{M}$ in DMF) and $100 \mu \mathrm{L}$ of DEHA were added to $10 \mathrm{~mL}$ of DMF and stirred at the room temperature for $10 \mathrm{~min}$ and then at $50{ }^{\circ} \mathrm{C}$ for $16 \mathrm{~h}$. The nanowires were recovered by centrifugation and washed with $\mathrm{H}_{2} \mathrm{O}$. The fraction of Pd in the nanowires (Pd-ls-x) was tuned by adjusting the concentration of $\operatorname{Pd}(\mathrm{acac})_{2}$ in the precursor. 
The Au-Ag alloy nanowires (thickness, $\sim 2.9 \mathrm{~nm}$ ) were synthesized by regrowth of the as-prepared Au-Ag alloy nanowires (thickness, $\sim 1.8 \mathrm{~nm}$ ). First, $20 \mathrm{~mL}$ of the Au-Ag alloy nanowires (thickness, $\sim 1.8$ $\mathrm{nm}$, as-synthesized without centrifugation and washing) were mixed with $4 \mathrm{~mL}$ of $\mathrm{PVP}\left(5 \mathrm{wt} \%\right.$ in $\left.\mathrm{H}_{2} \mathrm{O}\right)$, $12 \mathrm{~mL}$ of DMF, and $34 \mathrm{~mL}$ of $\mathrm{H}_{2} \mathrm{O}$. Then, $480 \mu \mathrm{L}$ of $\mathrm{HAuCl}_{4}\left(0.025 \mathrm{M}\right.$ in DMF) and $1.2 \mathrm{~mL}$ of $\mathrm{AgNO}_{3}$ $(0.01 \mathrm{M})$ were added successively into the above solution and stirred at the room temperature for $30 \mathrm{~min}$. The resulting Au-Ag alloy nanowires (thickness, $\sim 2.9 \mathrm{~nm}$ ) were redispersed in $15 \mathrm{~mL}$ of DMF as the seeds for synthesis of the Au-Ag-Pd alloy nanowires $\left(\mathrm{Pd}-l s^{\prime}-x\right)$. The fraction of $\mathrm{Pd}$ in the nanowires $\left(\mathrm{Pd}-l s^{\prime}-x\right)$ was tuned by adjusting the amount of $\operatorname{Pd}(\mathrm{acac})_{2}$ in the precursor.

Electrochemical measurements. Electrochemical measurements were carried out on CHI 760E electrochemical working station ( $\mathrm{CH}$ Instruments, Inc.) using a three-electrode configuration at room temperature $\left(25^{\circ} \mathrm{C}\right)$. A saturated calomel electrode (SCE) was used as the reference electrode. In evaluating the electrocatalytic activity and durability, a graphite rod was used as the counter electrode. The calibration of the reference electrode was performed in high-purity $\mathrm{H}_{2}$ saturated electrolyte with a $\mathrm{Pt}$ foil as the working electrode. All potentials are reported with respect to the reversible hydrogen electrode (RHE). The ultrathin Au-Ag-Pd alloy nanowires were supported on the carbon black (Vulcan XC-72) with a metal loading amount of $\sim 20 \%$ (the exact loading amount was affirmed by ICP-MS) and dispersed in a mixture of isopropanol, milli-Q water, and 5\% Nafion (volume ratio, 4:1:0.02). After ultrasonication for $1 \mathrm{~h}$, a homogeneous ink was obtained. The working electrode was prepared by depositing a known volume of the ink $(\mathrm{Pd}, 1 \mu \mathrm{g})$ on the polished surface of a rotating disk electrode $\left(\mathrm{RDE}, 0.196 \mathrm{~cm}^{2}\right)$ and dried at the room temperature. The commercial Pd/C (Innochem, $10 \mathrm{wt} \% \mathrm{Pd}$ ) was used as a benchmark for comparison.

All electrolytes were purged with high-purity $\mathrm{N}_{2}$ for at least $30 \mathrm{~min}$ before use. The working electrode was initially cycled between 0.1 and $1.2 \mathrm{~V}$ at a scan rate of $100 \mathrm{mV} \mathrm{s}^{-1}$ in $\mathrm{N}_{2}$-saturated $0.5 \mathrm{M} \mathrm{KOH}$ until a steady curve was obtained. The ECSA of the catalyst was measured according to the coulombic charges associated with the reduction of the $\mathrm{PdO}$ monolayer assuming a reference value of $4.05 \mathrm{C} \mathrm{m}^{-2} \cdot{ }^{2-3}$ To evaluate the activity of the catalysts in the alcohol oxidation reactions, CV curves were recorded between 0.1 and $1.2 \mathrm{~V}$ at a scan rate of $50 \mathrm{mV} \mathrm{s}^{-1}$ in different electrolytes (base + alcohol): (1) Ethylene glycol oxidation reaction (EGOR), 0.5 M KOH + 0.5 M EG; (2) Glycerol oxidation reaction (GOR), $1 \mathrm{M} \mathrm{KOH}$ 
$+0.1 \mathrm{M}$ glycerol; (3) Ethanol oxidation reaction (EOR), $1 \mathrm{M} \mathrm{KOH}+1 \mathrm{M} \mathrm{EtOH}$. To obtain the CO stripping voltammetry, the working electrode was held at $0.1 \mathrm{~V}$ for $30 \mathrm{~min}$ in CO-saturated $0.5 \mathrm{M} \mathrm{KOH}$ to achieve maximum coverage of $\mathrm{CO}$ at the $\mathrm{Pd}$ sites. $\mathrm{CO}$ was then removed from the electrolyte by purging with $\mathrm{N}_{2}$ for $30 \mathrm{~min}$. CV curves were collected in the potential range of $0.1-1.2 \mathrm{~V}$ at a scan rate of $50 \mathrm{mV}$ $\mathrm{s}^{-1}$.

Analysis of the EGOR products. The products of the electrocatalytic EGOR were analyzed by the high-performance liquid chromatography (HPLC). The column used was an xtimate Sugar-H column (5 $\mu \mathrm{m}, 7.8 \times 300 \mathrm{~mm}$ ), which was maintained at $70^{\circ} \mathrm{C}$ in a column oven during the analysis. Sulfuric acid $\left(\mathrm{H}_{2} \mathrm{SO}_{4}, \mathrm{pH} 2\right)$ was used as the mobile phase with a flow rate of $0.5 \mathrm{~mL} \mathrm{~min}^{-1}$. A refractive index detector (RID) was used for the analysis. The chronoamperometric $i-t$ test was performed at $0.8 \mathrm{~V} v s$. RHE in $\mathrm{N}_{2}-$ saturated $0.1 \mathrm{M} \mathrm{KOH}+0.5 \mathrm{M} \mathrm{EG}$ and stopped when the quantity of electricity reached $50 \mathrm{C}$. The electrolyte was then sampled and analyzed by the HPLC. The concentrations of the possible products, including oxalic acid, glyoxylic acid, glycolic acid, glycolaldehyde, and formic acid, were calculated according to the standard curves. Faradaic efficiency of each product was calculated as $(\mathrm{mnF}) / \mathrm{Q}$, where $F$ is the Faraday constant, $m$ is the quantity of the product (mol), and $n$ is the number of the transferred electrons per molecule of the product.

Characterizations. Transmission electron microscopy (TEM) was performed on a Hitachi HT-7700 at $100 \mathrm{kV}$. High-resolution TEM (HRTEM) and Energy-dispersive X-ray spectroscopy (EDS) elemental mapping were performed on a JEM-F200-TEM at $200 \mathrm{kV}$. X-ray diffraction (XRD) patterns were recorded on a Rigaku SmartLab powder X-ray diffractometer equipped with $\mathrm{Cu} \mathrm{K} \alpha$ radiation. Inductively coupled plasma mass spectrometry (ICP-MS) was performed on a PerkinElmer NexION 350D. X-ray photoelectron spectroscopy (XPS) was conducted on a Thermo Fisher ESCALAB Xi+ equipped with monochromatic $\mathrm{Al} / \mathrm{Mg} \mathrm{K} \alpha$ radiation. EDS was carried out on a Hitachi SU3500 scanning electron microscope at $15 \mathrm{kV}$. HPLC analysis was performed on Agilent 1260. 


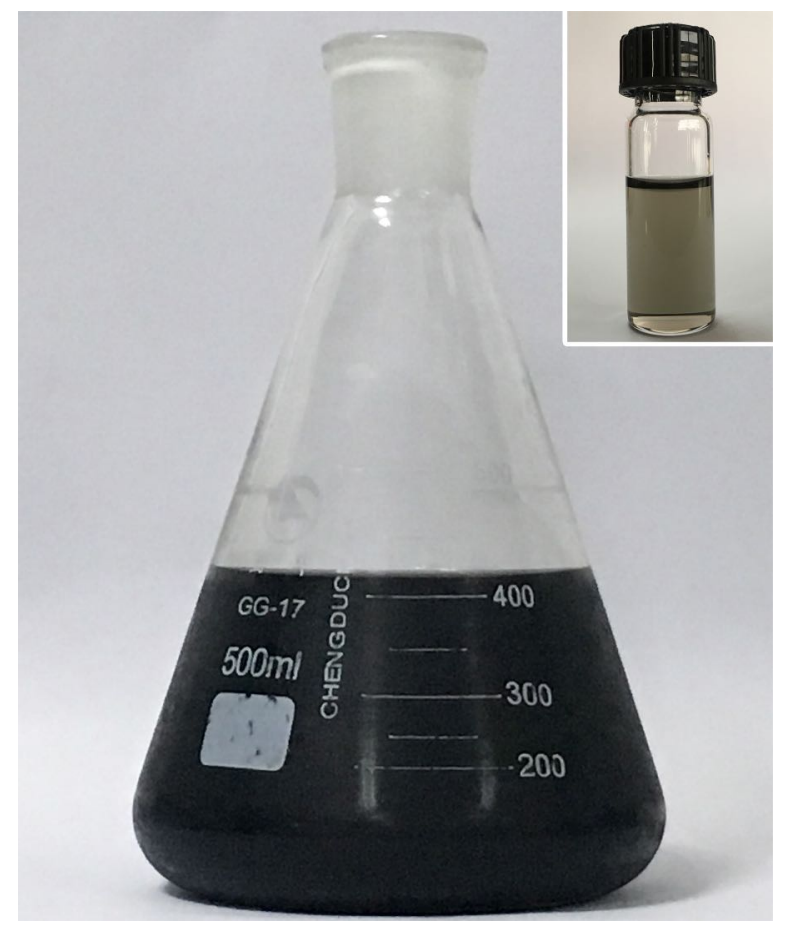

Figure S1. Lab-scale synthesis of the highly strained ultrathin Au-Ag-Pd alloy nanowires (Pd-hs-37.2\%) in a $500 \mathrm{ml}$-capacity flask. The typical synthesis in the experimental section was scaled up by 16 times, which produces $\sim 64 \mathrm{mg}$ of the highly strained ultrathin Au-Ag-Pd alloy nanowires supposing a yield of $100 \%$. This figure shows a photograph of a sol of the nanowires obtained from this synthesis. Inset: Sol of the nanowires after dilution. 

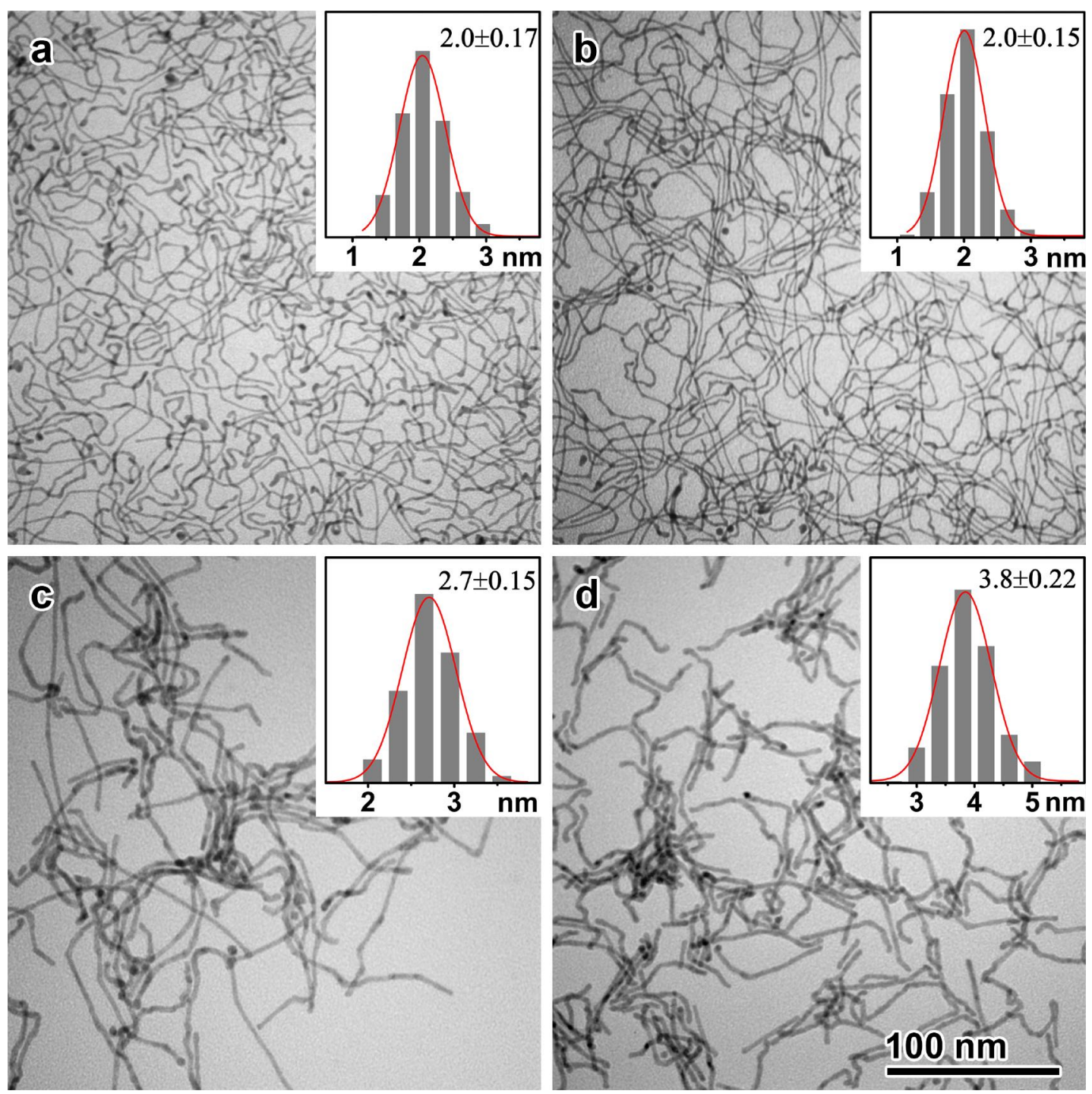

Figure S2. TEM images of the highly strained ultrathin Au-Ag-Pd alloy nanowires with different molar fractions of Pd (Pd-hs-x). (a-d) $x=12.2 \%, 26.0 \%, 54.1 \%$, and 71.4\%, respectively. Inset: Diameter histograms of the nanowires. The diameter increases from $\sim 2.0$ to $\sim 3.8 \mathrm{~nm}$ when the molar fraction of Pd increases from $12.2 \%$ to $71.4 \%$. 


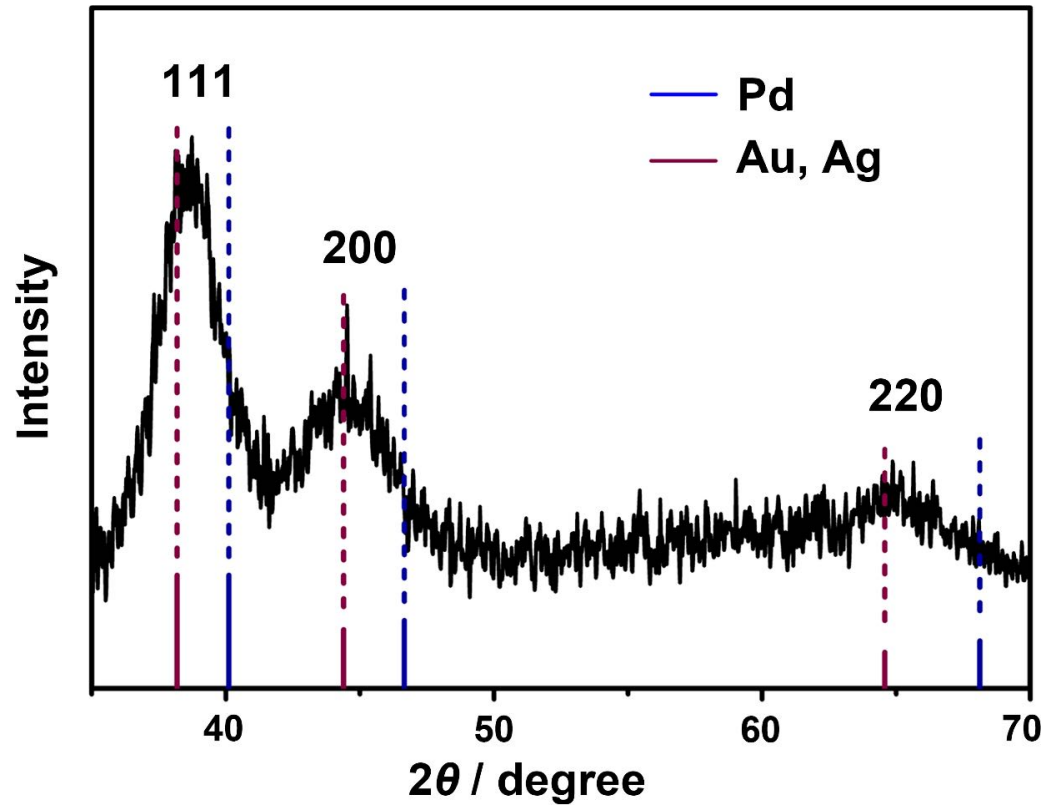

Figure S3. XRD pattern of the Pd- $h s-37.2 \%$ nanowires. The well-resolved peaks in the range of $30-70^{\circ}$ $2 \theta$ can be indexed as 111,200 , and 220 reflections, respectively. 


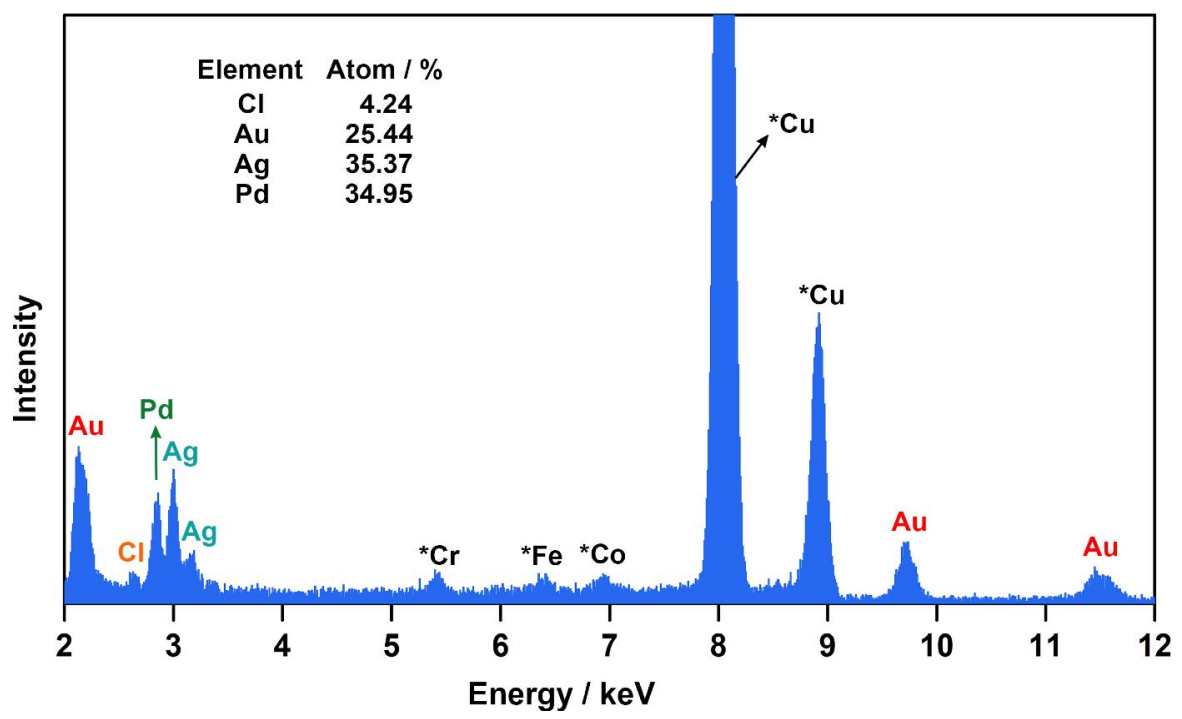

Figure S4. EDS spectrum of the highly strained ultrathin Au-Ag-Pd alloy nanowires (Pd-hs-37.2\%). Asterisks indicate the signals of interfering elements $(\mathrm{Cu}, \mathrm{Fe}, \mathrm{Co}, \mathrm{Cr})$ from the TEM grid, the sample holder, and the TEM pole shoe. 


\section{Role of each reagent in the formation of the ultrathin Au-Ag-Pd alloy nanowires:}

We hypothesize that the adsorption of $\mathrm{Cl}^{-}$preferentially at the $\mathrm{Ag}$ sites plays an important role in the formation of the ultrathin Au-Ag-Pd alloy nanowires. ${ }^{1}$ To verify it, control experiments were carried out to discriminate the roles of different reagents. An increase in the concentration of $\mathrm{Ag}^{+}$in the precursor led to a shift of the product from bulky faceted crystals to nanospheres and eventually ultrathin nanowires (Figure S5). An appropriate concentration of $\mathrm{Cl}^{-}$was also found necessary to avoid the formation of tadpole-like nanowires (Figure S6). Both results confirm that the Ag-Cl interaction passivates the lateral

faces of the nanowires and confines the crystal growth, which accounts for the one-dimensional shape and the ultrasmall thickness of the nanowires obtained from this synthesis. It is worth noting that this synthesis is particularly successful with $\mathrm{Cl}^{-}$. Spherical nanoparticles instead of the nanowires were obtained when $\mathrm{Br}^{-}$or $\mathrm{I}^{-}$was used in this synthesis (Figure S7). A plausible explanation is that $\mathrm{Br}^{-}$and $\mathrm{I}^{-}$bind at faces that are different from those that $\mathrm{Cl}^{-}$binds, leading to a difference in the morphology. Further control experiments (Figure S8-S10) suggest that DEHA serves as a reducing agent, and PVP serves as a surfactant to maintain the colloidal dispersity of the nanowires. The use of DMF rather than $\mathrm{H}_{2} \mathrm{O}$ as the solvent significantly slows down the reduction rate of the metal salts, which facilitates the controlled growth of the nanowires. ${ }^{1,4-5}$ 

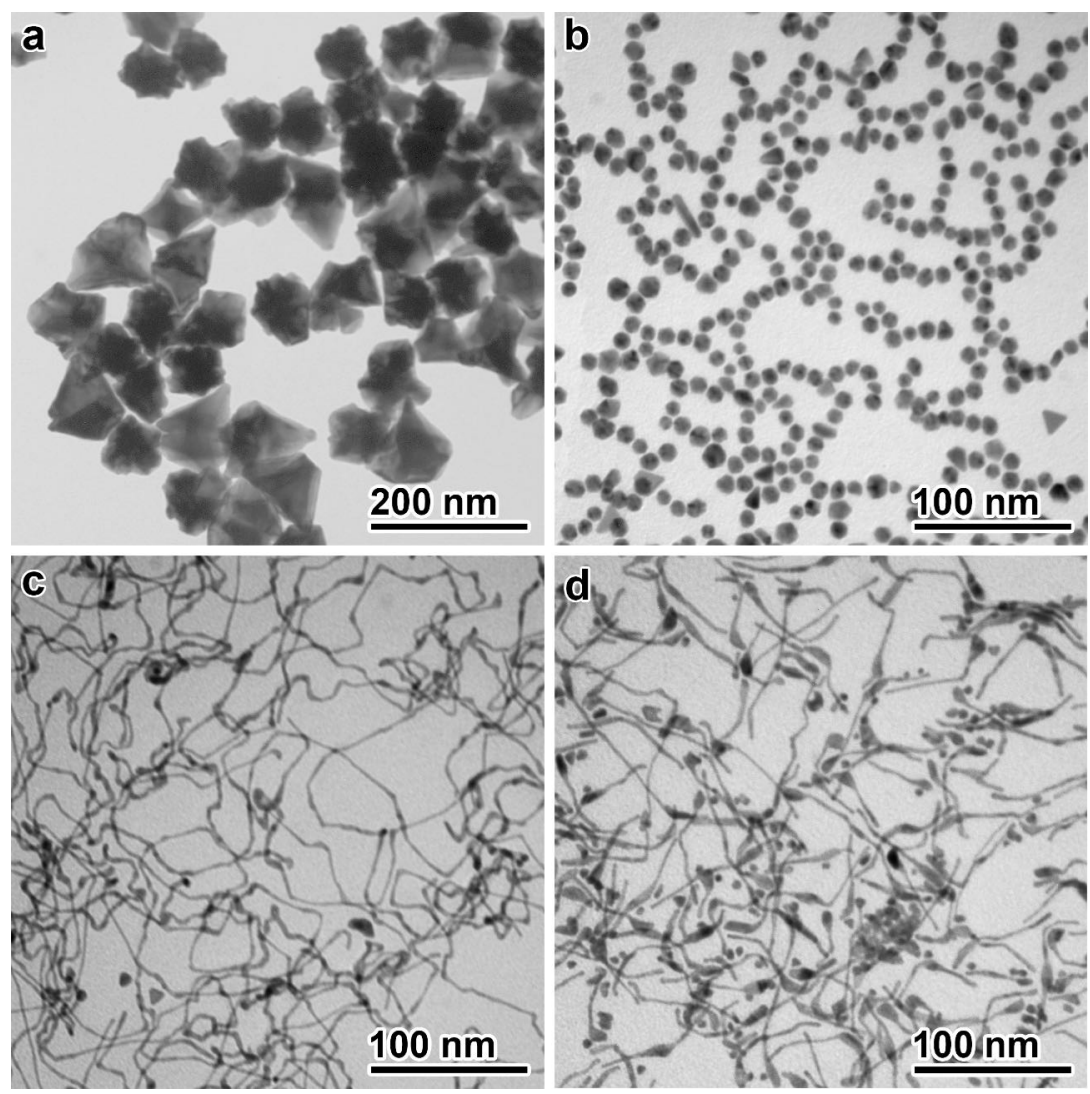

Figure S5. Role of Ag in the formation of the ultrathin Au-Ag-Pd alloy nanowires (Pd-hs-37.2\%). (a-d) TEM images of the products synthesized with a $\mathrm{AgNO}_{3} / \mathrm{HAuCl}_{4}$ ratio of $0,0.2,0.4$, and 2 , respectively. Discussion: In the absence of Ag, bulky nanocrystals $(\sim 100 \mathrm{~nm})$ instead of nanowires were obtained. When a small amount of $\mathrm{AgNO}_{3}$ was added to the synthesis system, small nanospheres $(\sim 10 \mathrm{~nm})$ were obtained, which can be attributed to the emergence of the surface $\mathrm{Ag}-\mathrm{Cl}$ bonds that limits the particle growth. A further increase in the concentration of $\mathrm{AgNO}_{3}$ led to the formation of the ultrathin nanowires, highlighting the role of the surface $\mathrm{Ag}-\mathrm{Cl}$ bonds in regulating the crystal growth. When the concentration of $\mathrm{AgNO}_{3}$ was too high, the thickness of the nanowires became nonuniform, resembling a tadpole shape, which can be attributed to the low $\mathrm{Cl}^{-} / \mathrm{Ag}$ ratio that weakens the surface passivation of the nanowires during the formation. Therefore, Ag plays a critical role in the formation of the ultrathin Au-Ag-Pd alloy nanowires. Its concentration should be carefully adjusted for the successful synthesis of the nanowires. 

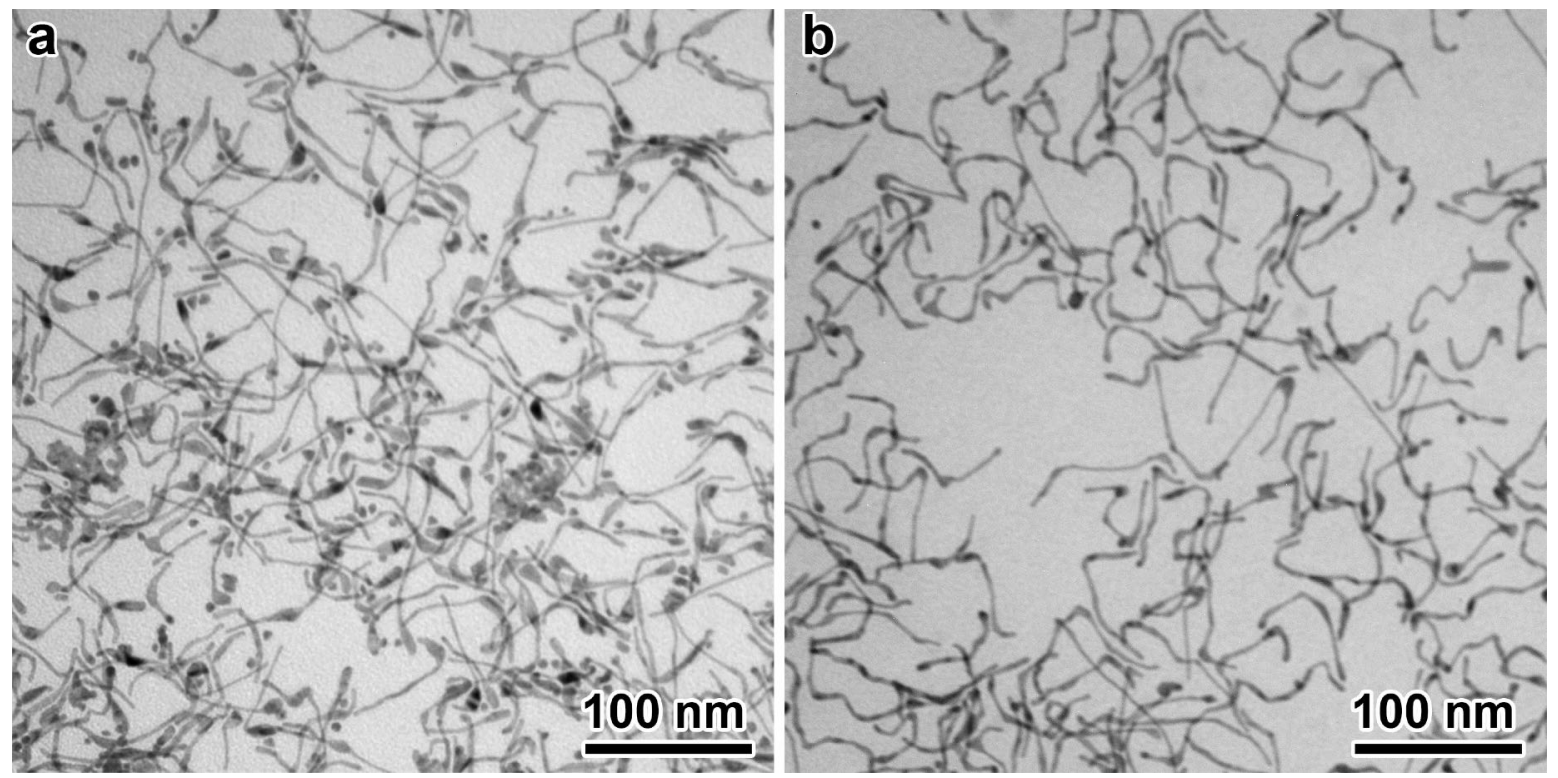

Figure S6. Role of $\mathrm{Cl}^{-}$in the formation of the ultrathin Au-Ag-Pd alloy nanowires (Pd-hs-37.2\%). (a) AuAg-Pd tadpole-shaped nanowires were obtained from a synthesis with a $\mathrm{AgNO}_{3} / \mathrm{HAuCl}_{4}$ ratio of 2. (b) Ultrathin Au-Ag-Pd alloy nanowires were obtained after adding extra $\mathrm{NaCl}$ into the synthesis $\left(\mathrm{Cl}^{-} / \mathrm{Ag}=\right.$ 5). Discussion: Tadpole-shaped Au-Ag-Pd nanowires were obtained with insufficient $\mathrm{Cl}^{-}$in the synthesis. In this case, the capping effect of the surface $\mathrm{Ag}-\mathrm{Cl}$ bonds were not fully established. When additional $\mathrm{Cl}^{-}$was added into the synthesis system, appropriate surface passivation was achieved, which gave rise to ultrathin Au-Ag-Pd alloy nanowires with improved uniformity in the thickness. 

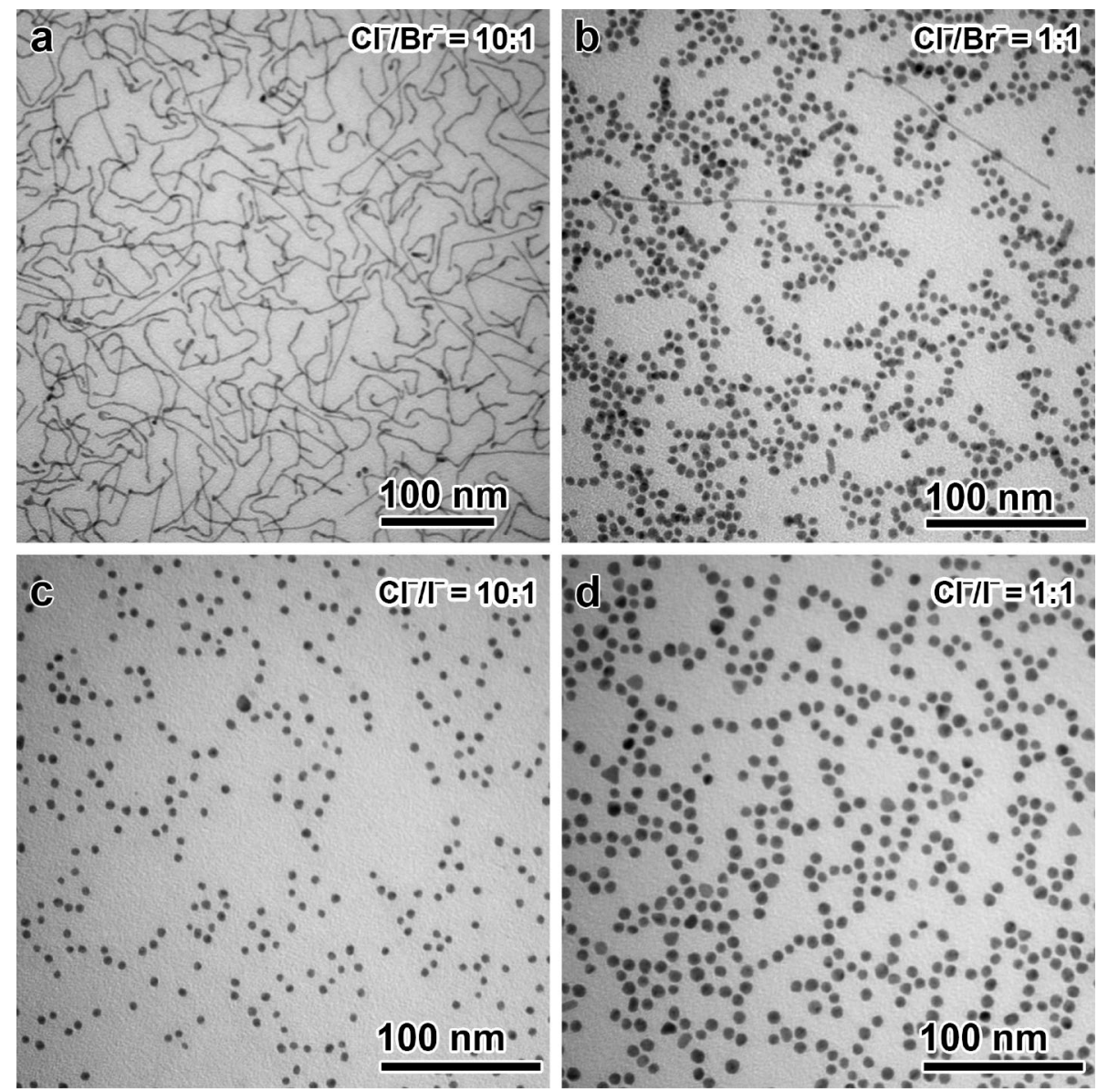

Figure S7. Effect of the halide on the morphology of the product. $\mathrm{Br}^{-}$or $\mathrm{I}^{-}$of different concentrations $\left(\mathrm{Cl}^{-}\right.$ $/ \mathrm{X}^{-}=10: 1$ or $\left.1: 1 ; \mathrm{X}=\mathrm{Br}, \mathrm{I}\right)$ were added to the synthetic system of the Au-Ag-Pd alloy nanowires (Pd$h s-37.2 \%)$. It can be inferred that the addition of $\mathrm{Br}^{-}$or $\mathrm{I}^{-}$led to the formation of spherical nanoparticles instead of nanowires. In particular, the change of the morphology occurred at a much lower concentration of $\mathrm{I}^{-}$than $\mathrm{Br}^{-}$. A plausible explanation is that $\mathrm{Br}^{-}$or $\mathrm{I}^{-}$binds at faces that are different from those that $\mathrm{Cl}^{-}$ binds, which accounts for the different morphology of the products. 

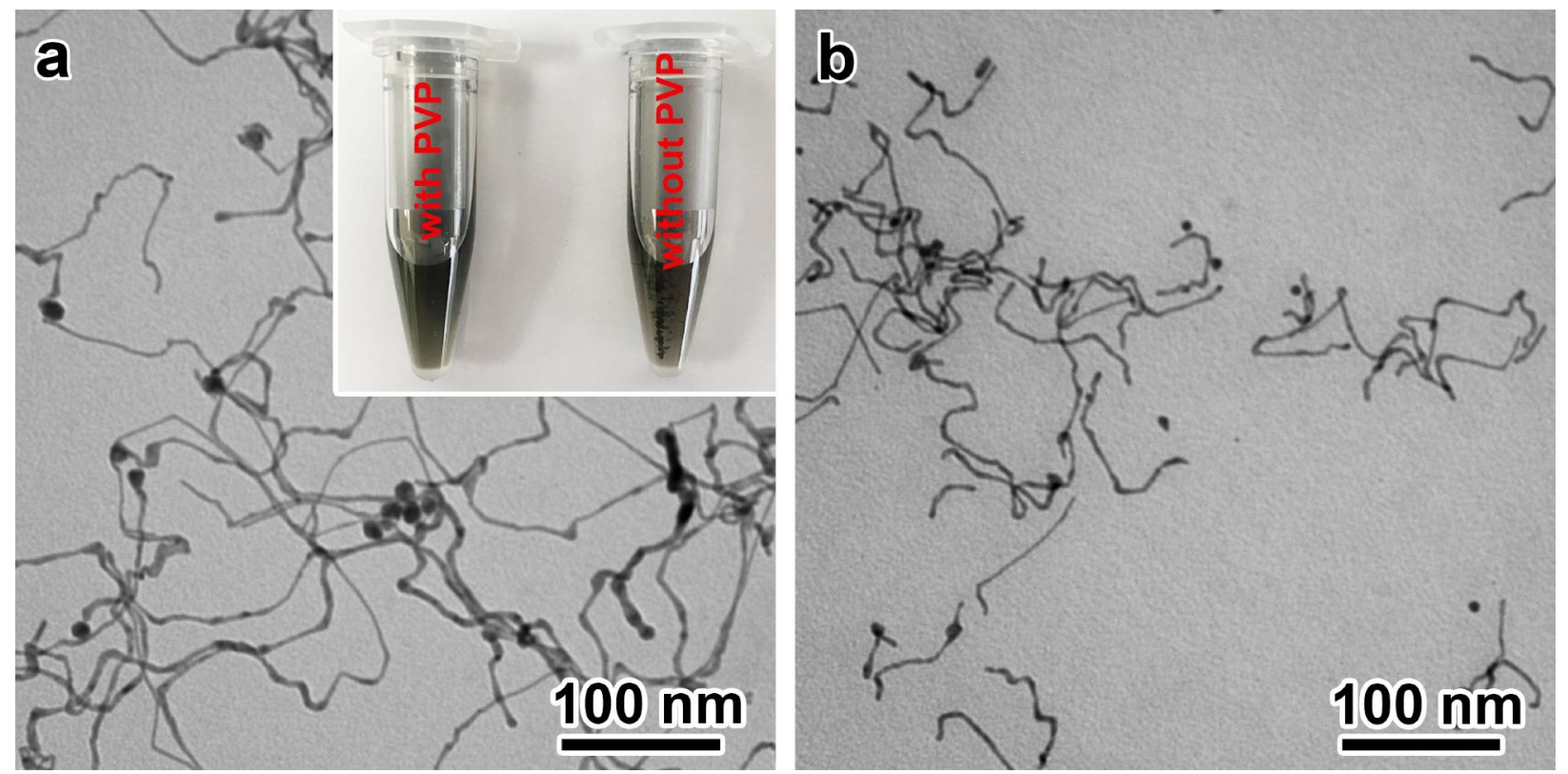

Figure S8. Role of PVP in the formation of the ultrathin Au-Ag-Pd alloy nanowires. The products were obtained from a modified synthesis of the Pd- $h s-37.2 \%$ nanowires. (a) No PVP was added to the synthetic system. (b) The concentration of PVP was 2.5 times greater than that in the typical synthesis. Discussion: PVP mainly serves as a dispersant of the nanowires. A synthesis in the absence of PVP gave rise to aggregated nanowires (see the photograph in the inset of a), and that in the excess of PVP gave rise to well-dispersed nanowires without significant change in the morphology. The successful formation of ultrathin nanowires in the absence of PVP further suggests that the one-dimensional growth of the nanowires does not rely on the capping effect of PVP. 

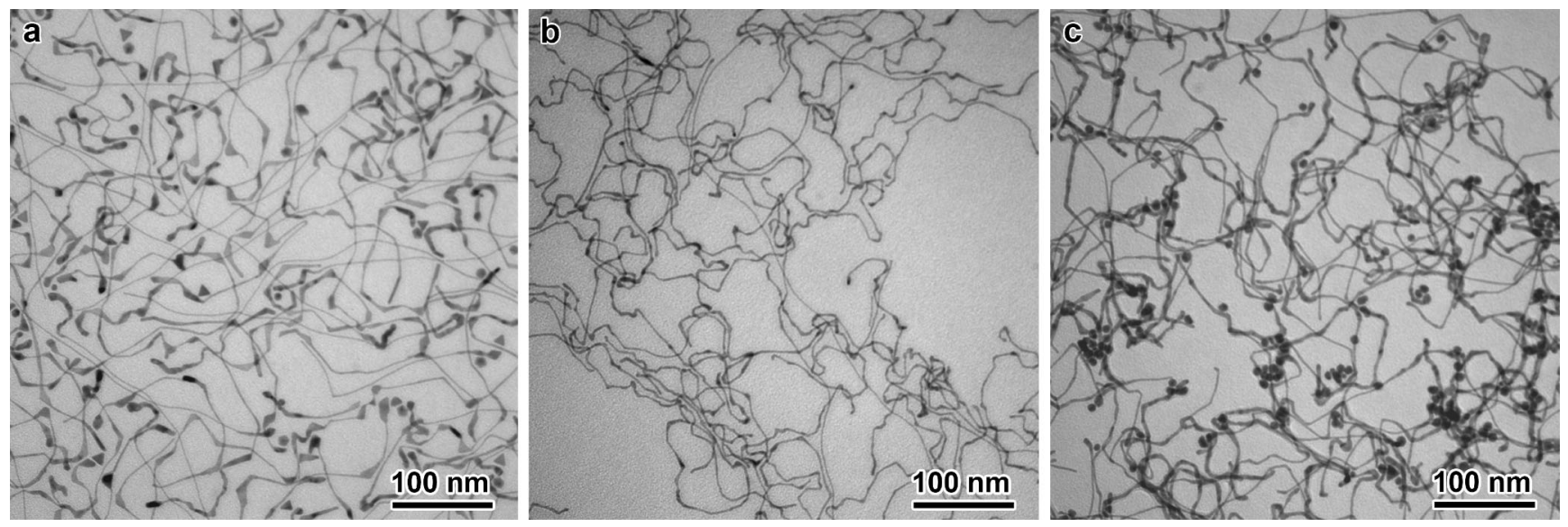

Figure S9. Role of DEHA in the formation of the ultrathin Au-Ag-Pd alloy nanowires. The products were obtained from a modified synthesis of the Pd- $h s-37.2 \%$ nanowires. (a) The synthesis was carried out in the absence of DEHA. To compensate, ascorbic acid was used as a reducing agent. (b) The concentration of DEHA was 0.25 times that in a typical synthesis. (c) The concentration of DEHA was 4 times greater than that of a typical synthesis. Discussion: DEHA serves as a reducing agent in the synthesis. When it was replaced by ascorbic acid in the synthesis, nanowires were still obtained in a high yield, suggesting that the one-dimensional growth of the nanowires does not rely on DEHA (a). When it was used in large excess, the reaction rate was greatly accelerated (according to the color change of the reaction solution), which led to free nucleation and thus formation of nanoparticles as impurities (c). 

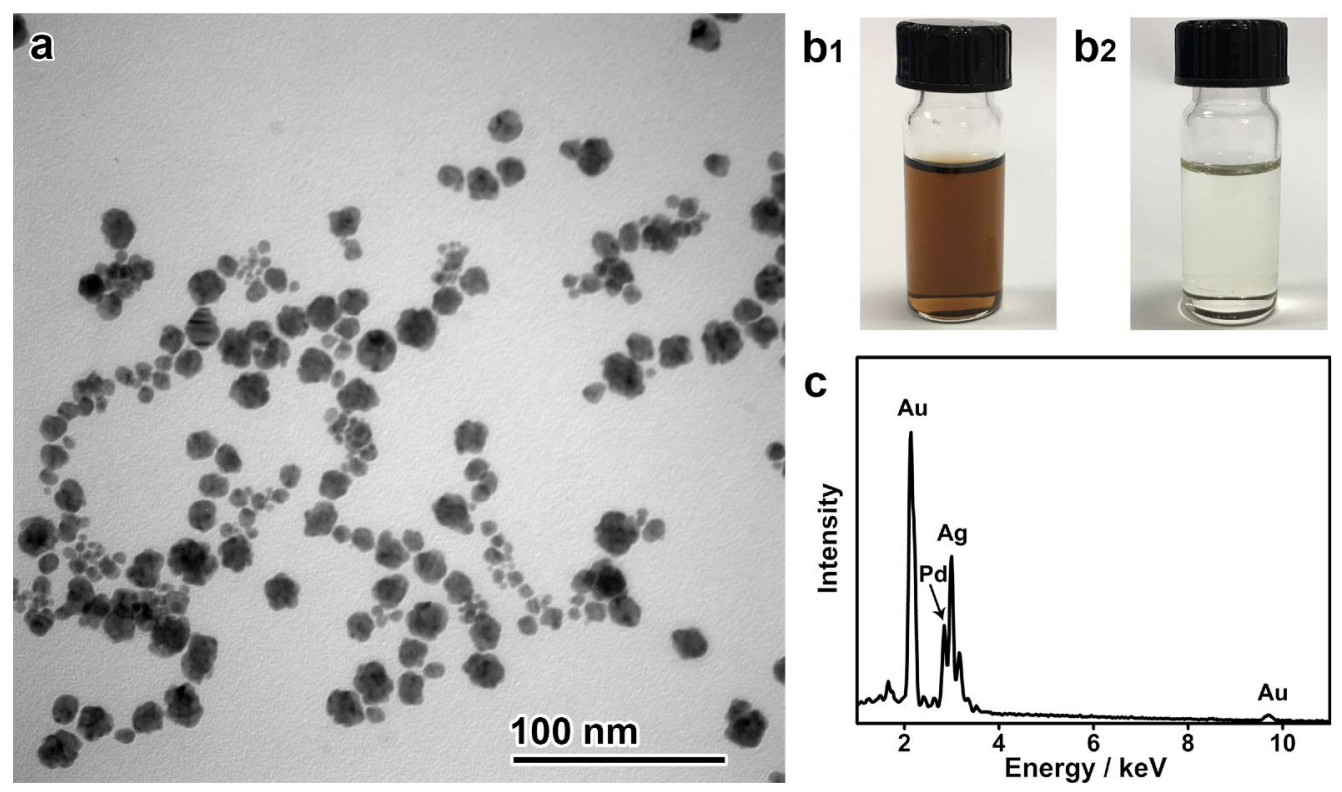

Figure S10. Role of DMF in the formation of the ultrathin Au-Ag-Pd alloy nanowires (Pd-hs-37.2\%). (a) TEM image of the product obtained from a typical synthesis using $\mathrm{H}_{2} \mathrm{O}$ as a solvent in place of DMF. (b $b_{1-}$ $b_{2}$ ) Photographs of the products obtained from a typical synthesis when all metal precursors, reducing agents, and surfactants were added to different solvents $\left(b_{1}, \mathrm{H}_{2} \mathrm{O} ; \mathrm{b}_{2}, \mathrm{DMF}\right)$ and stirred for 5 min in an ice-water bath. While the reaction conducted in $\mathrm{H}_{2} \mathrm{O}$ already gave rise to a dark-brown solution, reaction conducted in DMF gave rise to a light-yellow solution, suggesting a much sluggish reduction of the metal salts in DMF. (c) EDS spectrum of the product obtained from the synthesis conducted in $\mathrm{H}_{2} \mathrm{O}$, confirming the formation of a Au-Ag-Pd ternary noble metal nanoparticles. Discussion: The use of DMF rather than $\mathrm{H}_{2} \mathrm{O}$ as the solvent significantly slows down the reduction rate of the metal salts, which facilitates a controlled crystal growth at the two ends of each nanowire. 


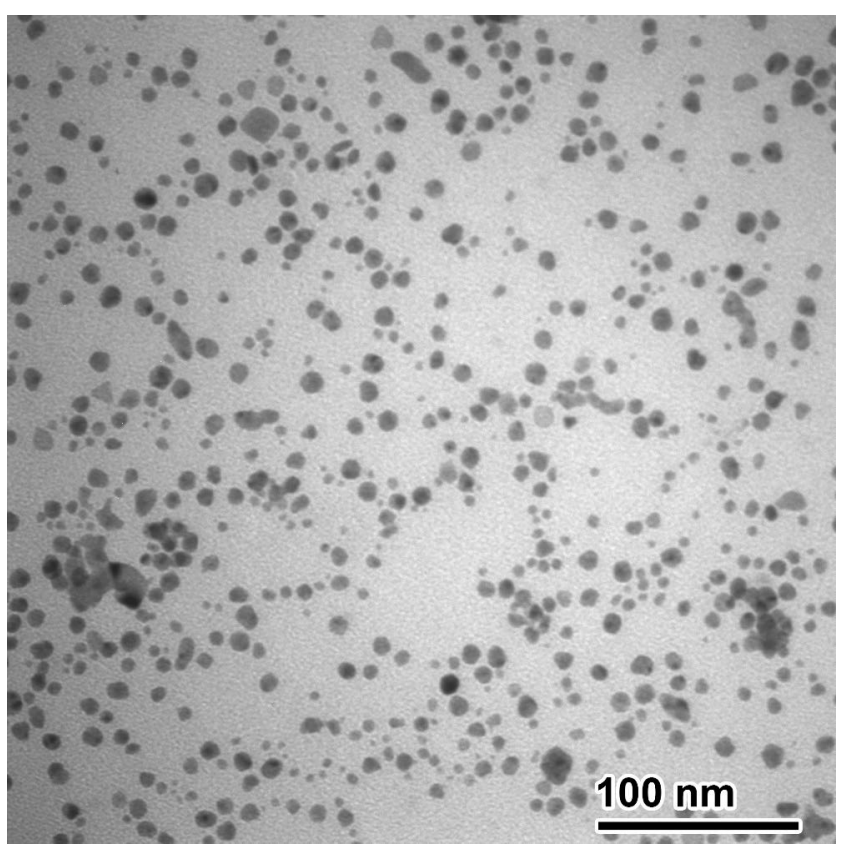

Figure S11. Role of $\mathrm{Au}$ in the formation of the ultrathin $\mathrm{Au}-\mathrm{Ag}-\mathrm{Pd}$ alloy nanowires (Pd-hs-37.2\%). The product was synthesized in the absence of $\mathrm{HAuCl}_{4}$. Nanoparticles were obtained instead of nanowires. We propose that $\mathrm{Ag}$ and $\mathrm{Pd}$ are less stable than $\mathrm{Au}$ when they are exposed to $\mathrm{DMF}, \mathrm{Cl}^{-}$, and ambient air $\left(\mathrm{O}_{2}\right)$. An oxidative etching may occur, as observed in the case of Ag in our previous work. ${ }^{6}$ Therefore, Au may stabilize the ultrathin nanowires in this synthesis against oxidative etching. 

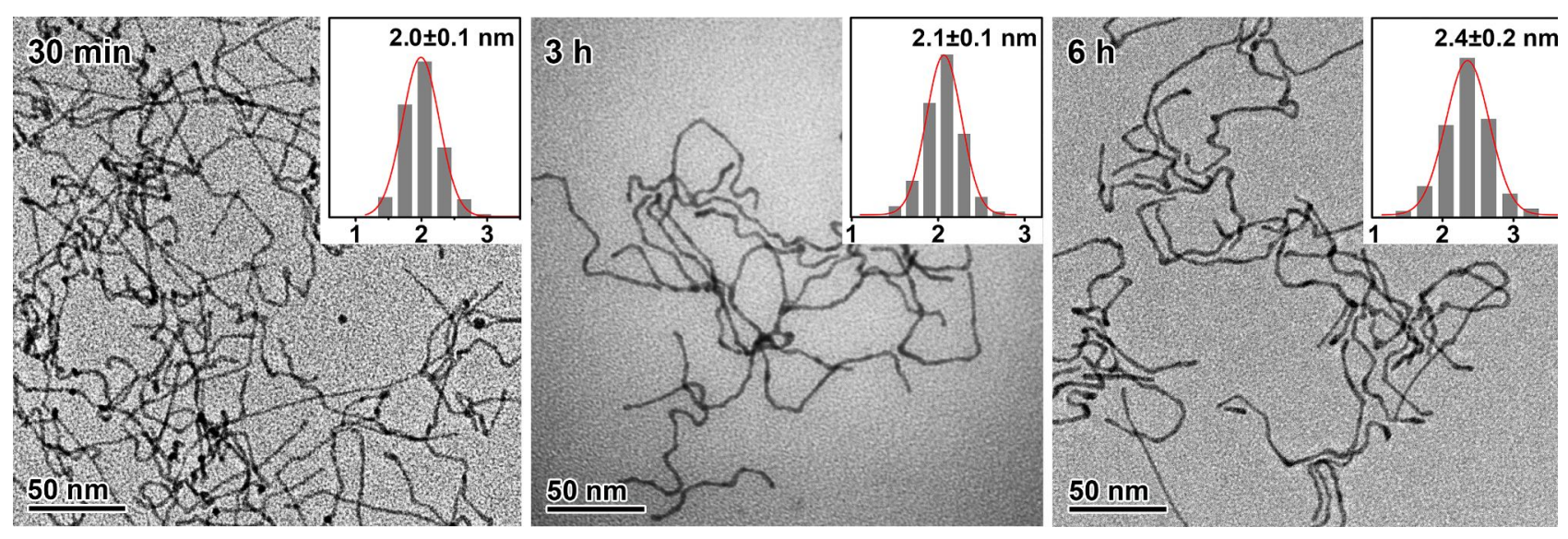

Figure S12. TEM images of intermediates obtained at $30 \mathrm{~min}, 3 \mathrm{~h}$, and $6 \mathrm{~h}$ from a typical synthesis of the Pd-hs-37.2\% nanowires. 

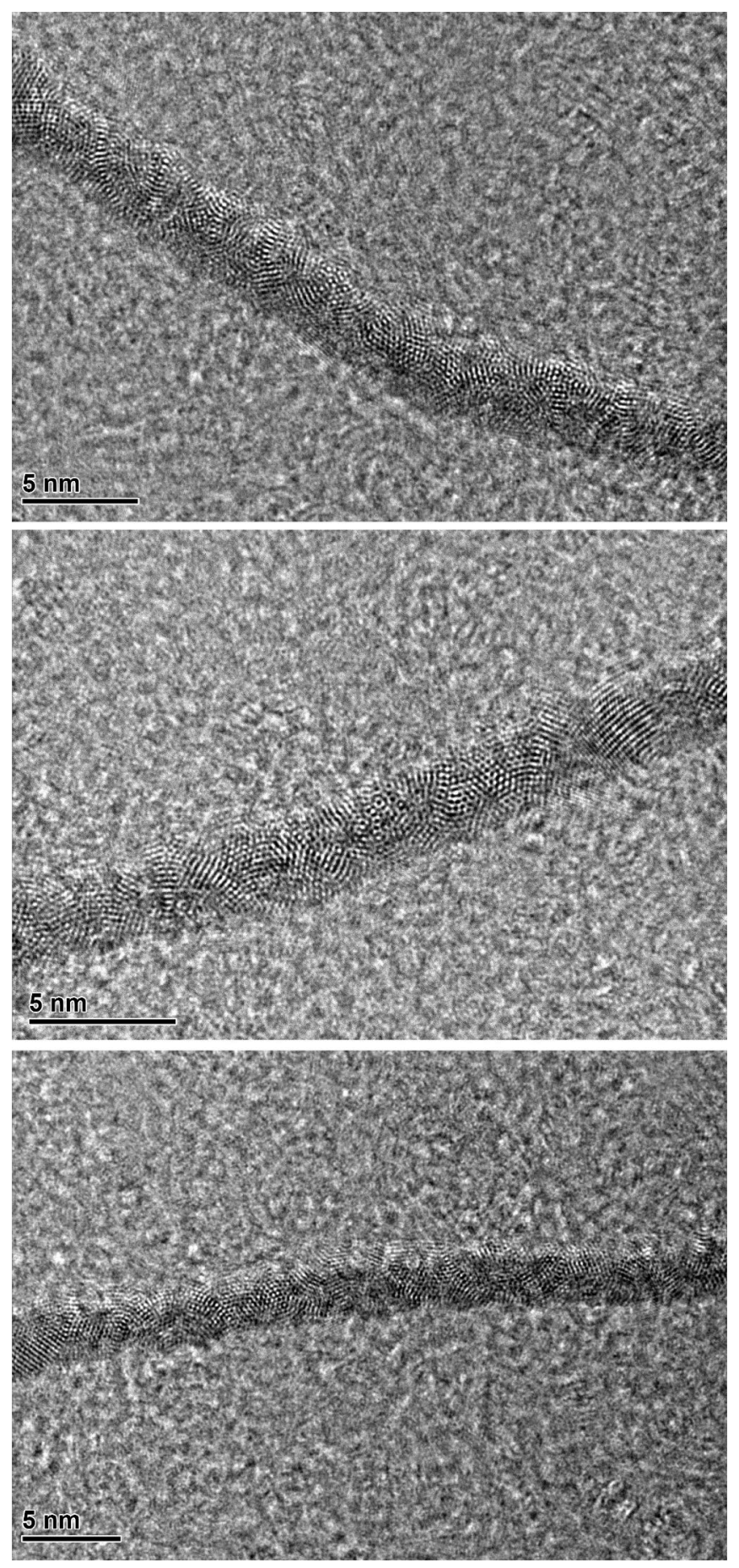

Figure S13. HRTEM images of the highly strained ultrathin Au-Ag-Pd alloy nanowires (Pd-hs-37.2\%). 

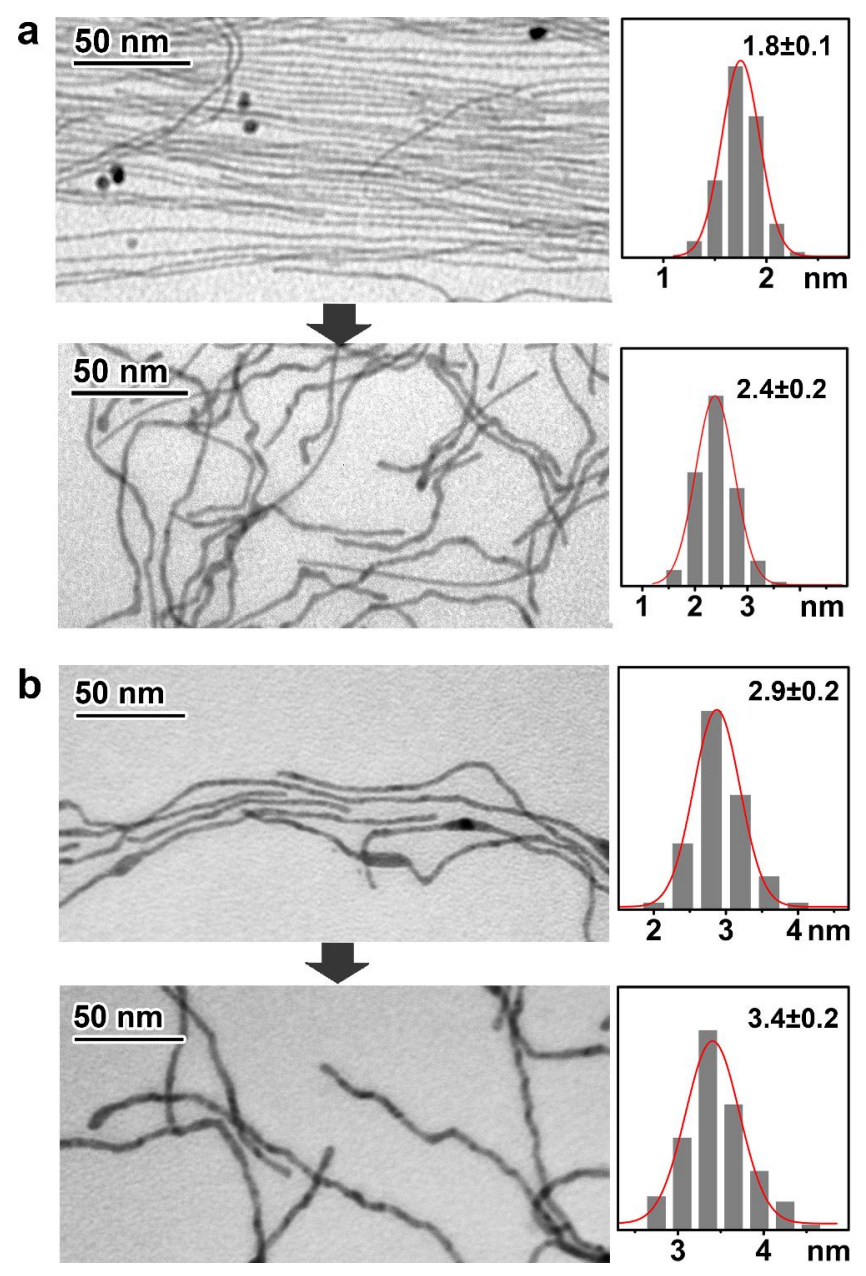

Figure S14. Synthesis of the less strained Au-Ag-Pd alloy nanowires (Pd-ls-28.1\%, Pd-ls'-25.1\%). (a) TEM images of the Au-Ag (top, average diameter: $1.8 \mathrm{~nm}$ ) and the Au-Ag-Pd alloy nanowires (bottom, Pd- $l s-28.1 \%$ ) before and after the Pd growth, respectively. (b) TEM images of the Au-Ag (top, average diameter: $2.9 \mathrm{~nm}$ ) and the $\mathrm{Au}-\mathrm{Ag}-\mathrm{Pd}$ alloy nanowires (bottom, Pd-ls'-25.1\%) before and after the Pd growth, respectively. Right to the images: Average diameters of the nanowires (mean \pm standard deviation). 

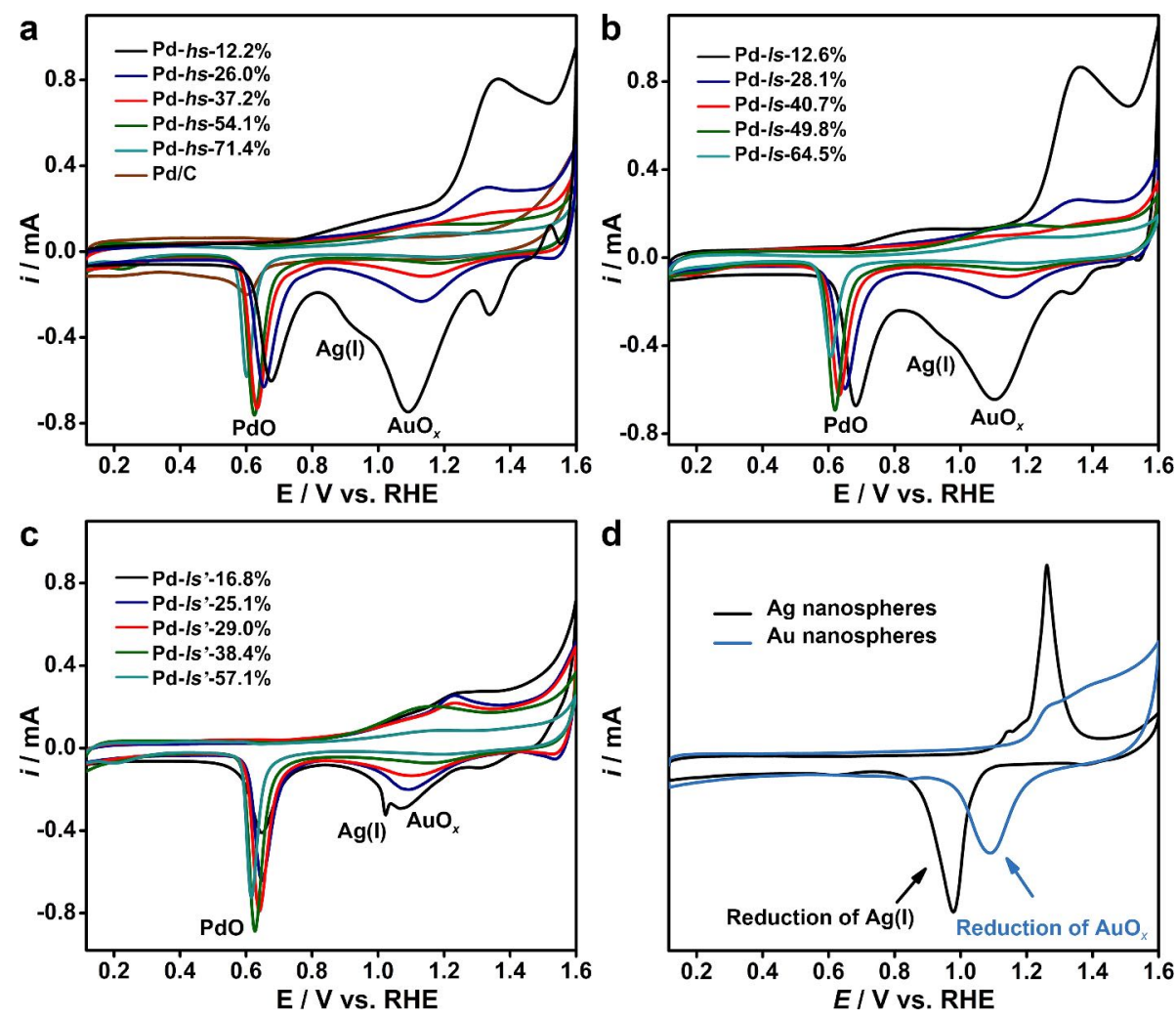

Figure S15. (a-c) CV curves of the highly strained Au-Ag-Pd alloy nanowires (Pd-hs-x), the less strained Au-Ag-Pd alloy nanowires ( $\mathrm{Pd}-l s-x$ and $\left.\mathrm{Pd}-l s^{\prime}-x\right)$, and the commercial $\mathrm{Pd} / \mathrm{C}$ in a potential range of $0.116-$ 1.6 $\mathrm{V}$ in $\mathrm{N}_{2}$-saturated $0.5 \mathrm{M} \mathrm{KOH}$ at $25^{\circ} \mathrm{C}$. Scan rate, $50 \mathrm{mV} \mathrm{s}^{-1}$. The peaks in the cathodic branch of the $\mathrm{CV}$ curves correspond to the reduction of $\mathrm{AuO}_{x}, \mathrm{Ag}(\mathrm{I})$, and $\mathrm{PdO}$, respectively. (d) $\mathrm{CV}$ curves of monometallic $\mathrm{Au}$ and $\mathrm{Ag}$, indicating the positions of the reduction peaks of the $\mathrm{AuO}_{x}$ and the $\mathrm{Ag}(\mathrm{I})$ species, respectively. It confirms that the surfaces of both the highly strained and the less strained Au-Ag-Pd alloy nanowires are composed of a $\mathrm{Au}-\mathrm{Ag}-\mathrm{Pd}$ alloy. 

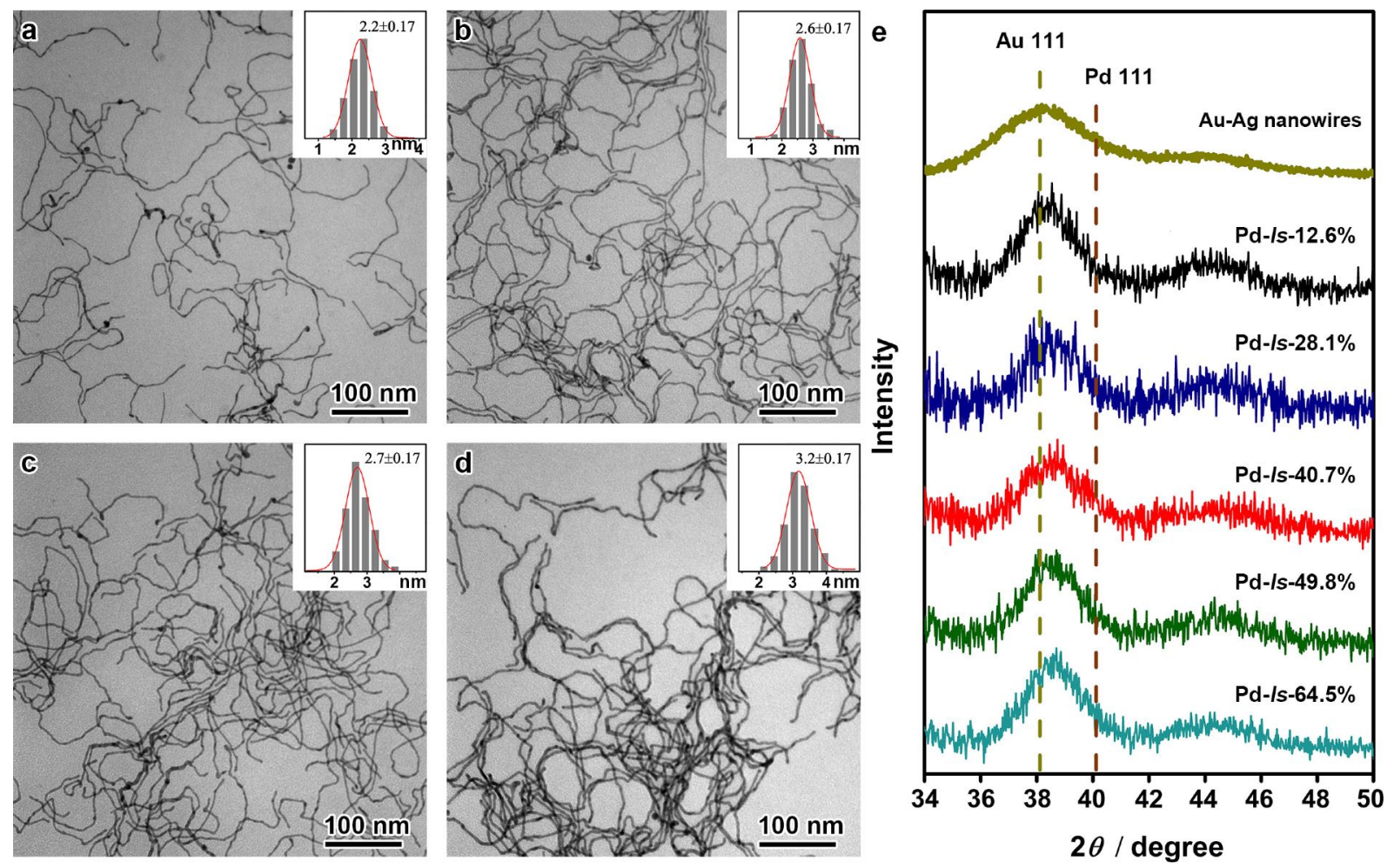

Figure S16. TEM images of the less strained ultrathin Au-Ag-Pd alloy nanowires with different molar fractions of $\mathrm{Pd}(\mathrm{Pd}-l s-x)$. (a-d) $x=12.6 \%, 40.7 \%, 49.8 \%$, and 64.5\%, respectively. Inset: Diameter histograms of the nanowires. The diameters of the nanowires increase from $\sim 2.2$ to $\sim 3.2 \mathrm{~nm}$ when the molar fractions of $\mathrm{Pd}$ increase from $12.6 \%$ to $64.5 \%$, indicating the radial growth of $\mathrm{Pd}$ on the ultrathin Au-Ag alloy nanowires. (b) XRD patterns of the Pd- $l s-x$ nanowires. The positions of the reflection peaks are between those of the monometallic $\mathrm{Pd}$ and $\mathrm{Au}$ (or $\mathrm{Ag}$ ), due to the $\mathrm{Au}-\mathrm{Ag}-\mathrm{Pd}$ alloying. 

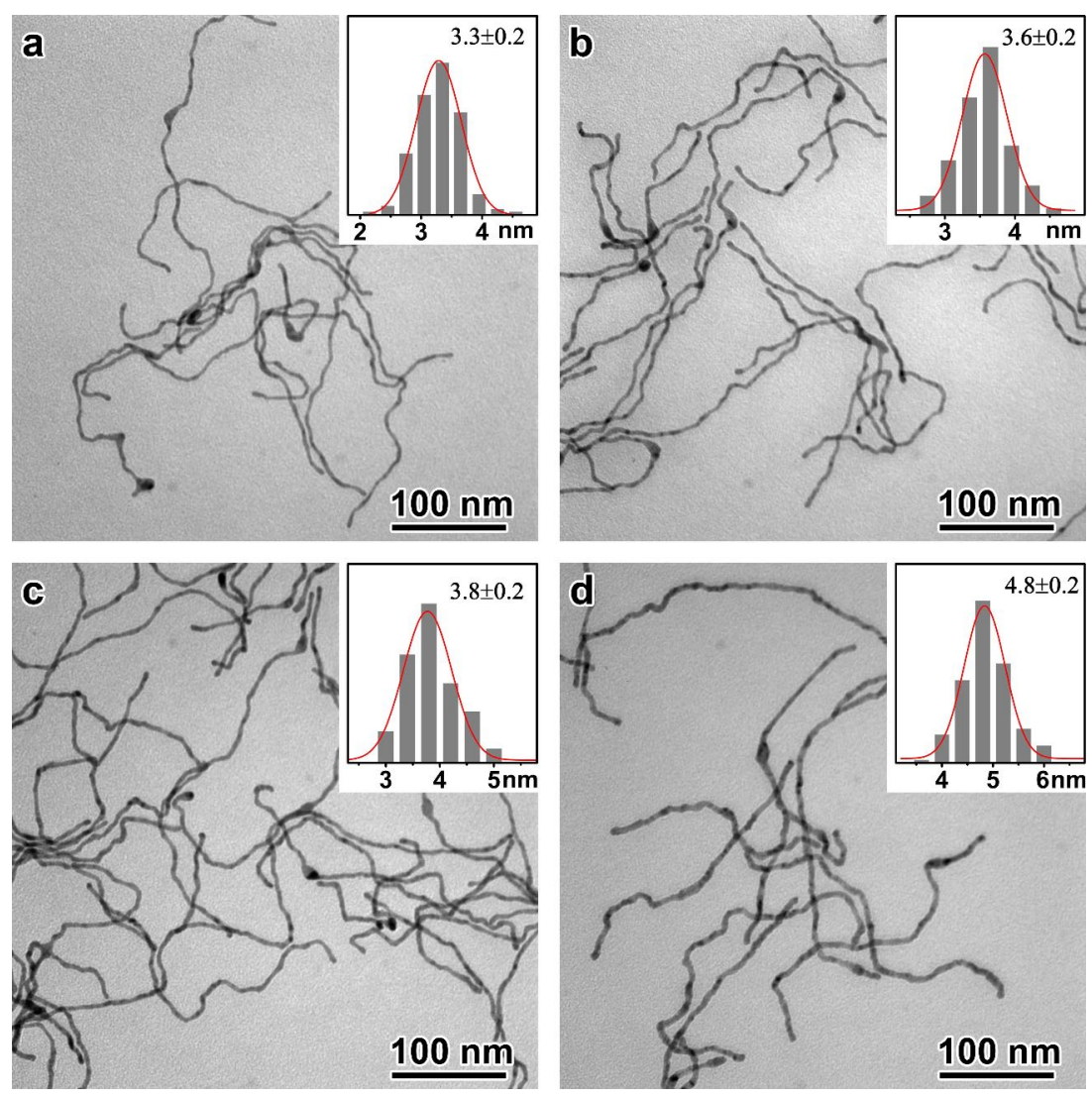

Figure S17. TEM images of the less strained ultrathin Au-Ag-Pd alloy nanowires (Pd- $l s$ ' $-x)$ with different molar fractions of $\mathrm{Pd}$. (a-d) $x=16.8 \%, 29.0 \%, 38.4 \%$, and $57.1 \%$, respectively. Inset: Diameter histograms of the nanowires. 


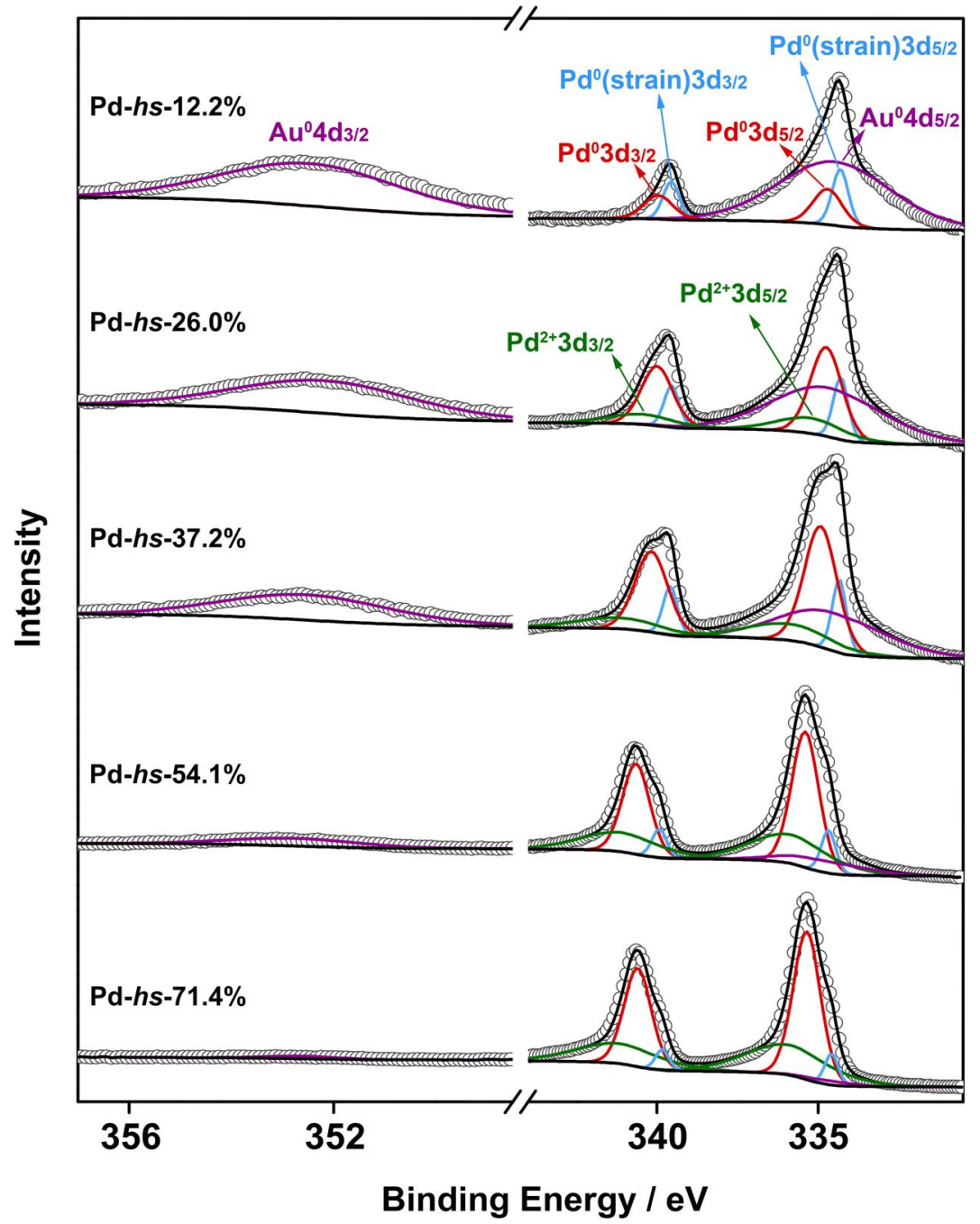

Figure S18. Core-level Pd 3d and Au 4d XPS spectra of the ultrathin Au-Ag-Pd alloy nanowires (Pd- $h s$ $x$ ). The $\mathrm{Au} 4 \mathrm{~d}$ XPS spectra were fitted to a pair of $4 \mathrm{~d}_{5 / 2}$ and $4 \mathrm{~d}_{3 / 2}$ doublets at $\sim 334$ and $352 \mathrm{eV}$, respectively. The intensity of the Au 4d XPS peaks decreases with increasing molar fraction of Pd. The Pd 3d XPS spectra were fitted to three symmetrical peaks at $\sim 334,335$, and $336 \mathrm{eV}$, which were assigned to $\mathrm{Pd}^{0}$ (strain), $\mathrm{Pd}^{0}$ and $\mathrm{Pd}^{2+}$ species, respectively. Both the $\mathrm{Pd}^{0}$ (strain) and $\mathrm{Pd}^{0}$ peaks shift to lower binding energies with decreasing fraction of $\mathrm{Pd}$ in the alloy nanowires, suggesting an increasing $\mathrm{Au}, \mathrm{Ag} \rightarrow \mathrm{Pd}$ electron transfer. With decreasing fraction of $\mathrm{Pd}$, the area ratio of $\mathrm{Pd}^{0}($ strain $) / \mathrm{Pd}^{0}$ increases monotonically (plot, see Figure 3a), which is in line with the increasing tensile strains in the nanowires. 

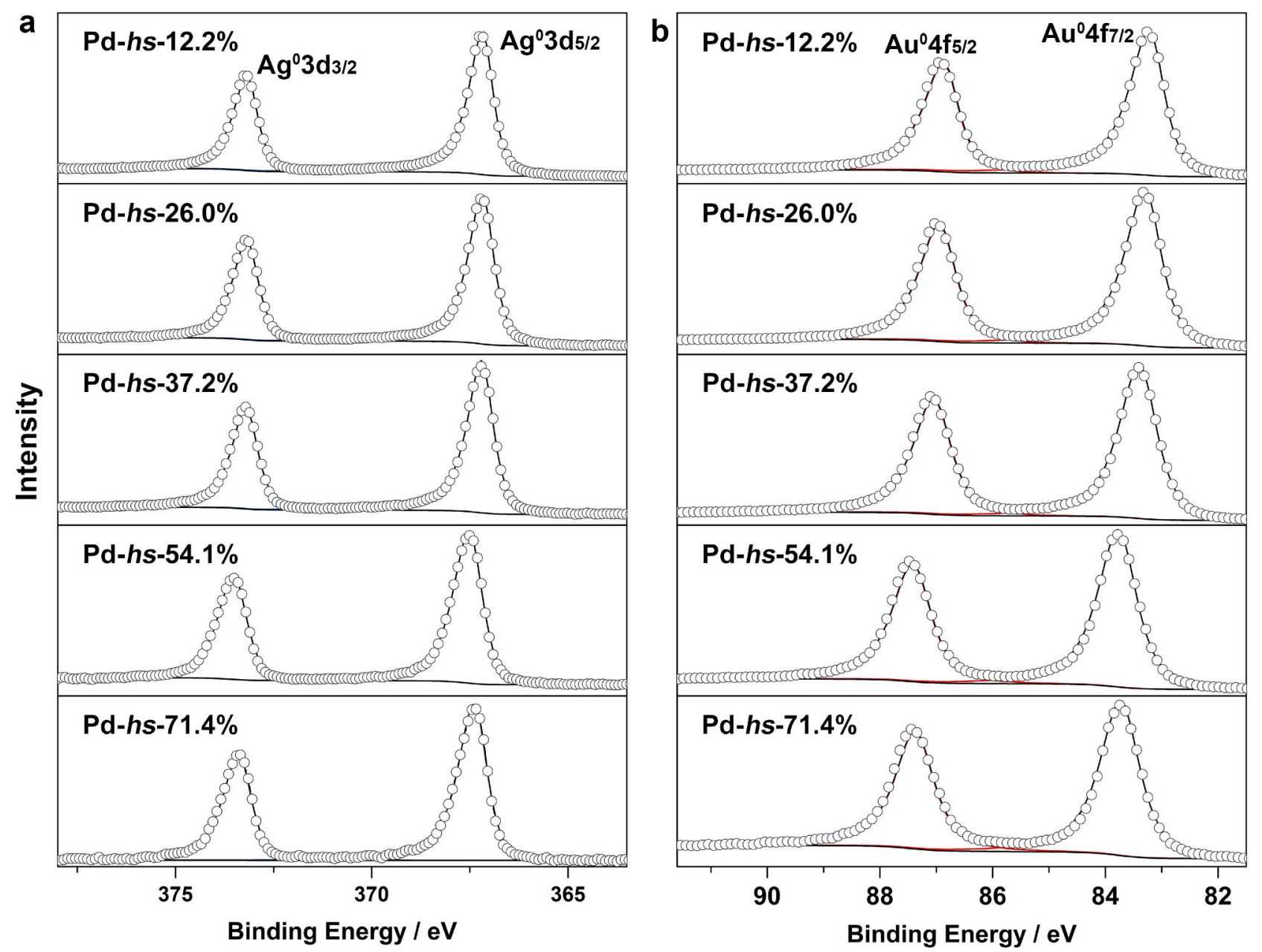

Figure S19. Core-level Ag 3d and Au 4f XPS spectra of the ultrathin Au-Ag-Pd alloy nanowires (Pd-hs$x$ ). The Ag $3 d$ XPS spectra can be fitted by $3 d_{5 / 2}$ and $3 d_{3 / 2}$ doublets, and the Au $4 f$ XPS spectra can be fitted by $4 \mathrm{f}_{7 / 2}$ and $4 \mathrm{f}_{5 / 2}$ doublets. Discussion: With an increasing fraction of $\mathrm{Pd}$, the $\mathrm{Au} 4 \mathrm{f}$ spectra shift continuously to higher binding energies, which suggests an electron transfer from Au to Pd. Because the Ag 3d XPS is not sensitive to its oxidation state, no significant shift of the spectra can be observed. Due to the electronegativity, it is reasonable that an electron transfer from $\mathrm{Ag}$ to $\mathrm{Pd}$ is also favorable. 


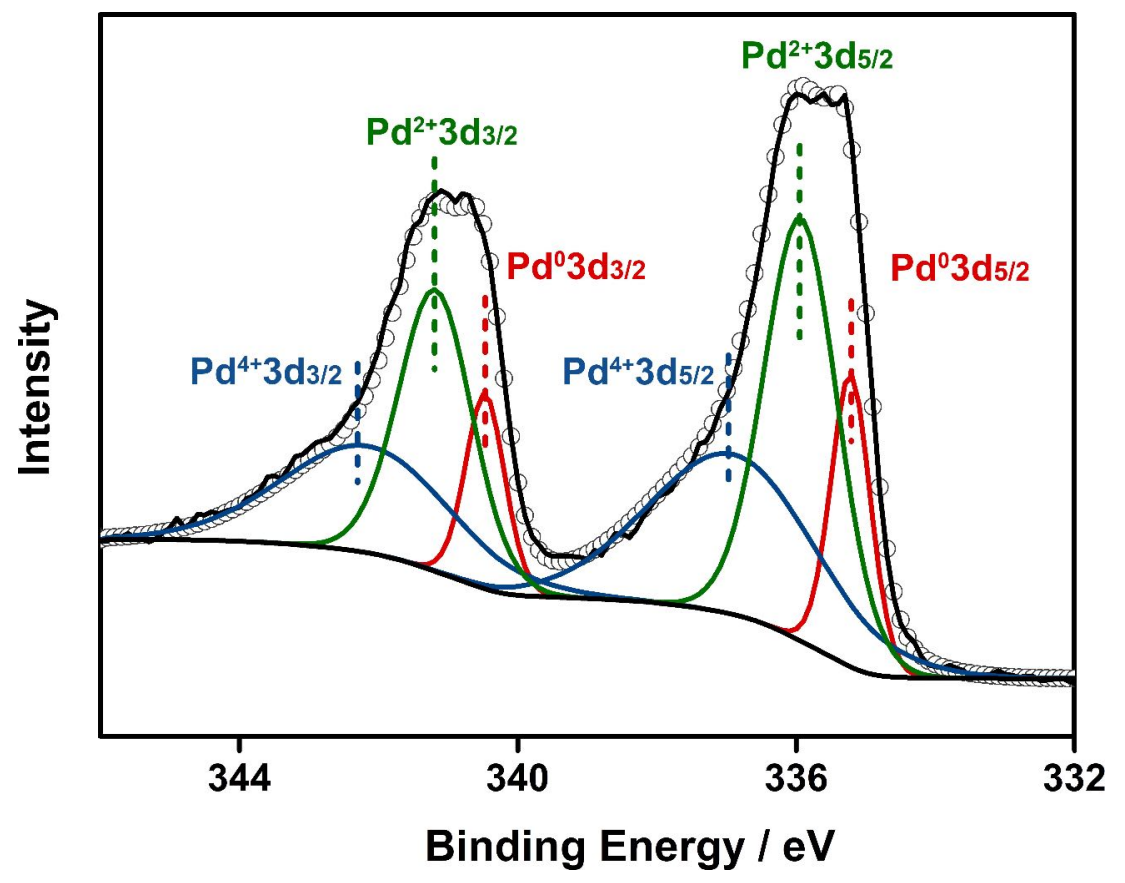

Figure S20. Core-level Pd 3d XPS of the commercial Pd/C (Innochem, $10 \mathrm{wt} \% \mathrm{Pd}$ ). The peaks were fitted with three symmetrical components at $\sim 335,336$, and $337 \mathrm{eV}$, which could be assigned to $\mathrm{Pd}^{0}, \mathrm{Pd}^{2+}$ and $\mathrm{Pd}^{4+}$ species, respectively. 

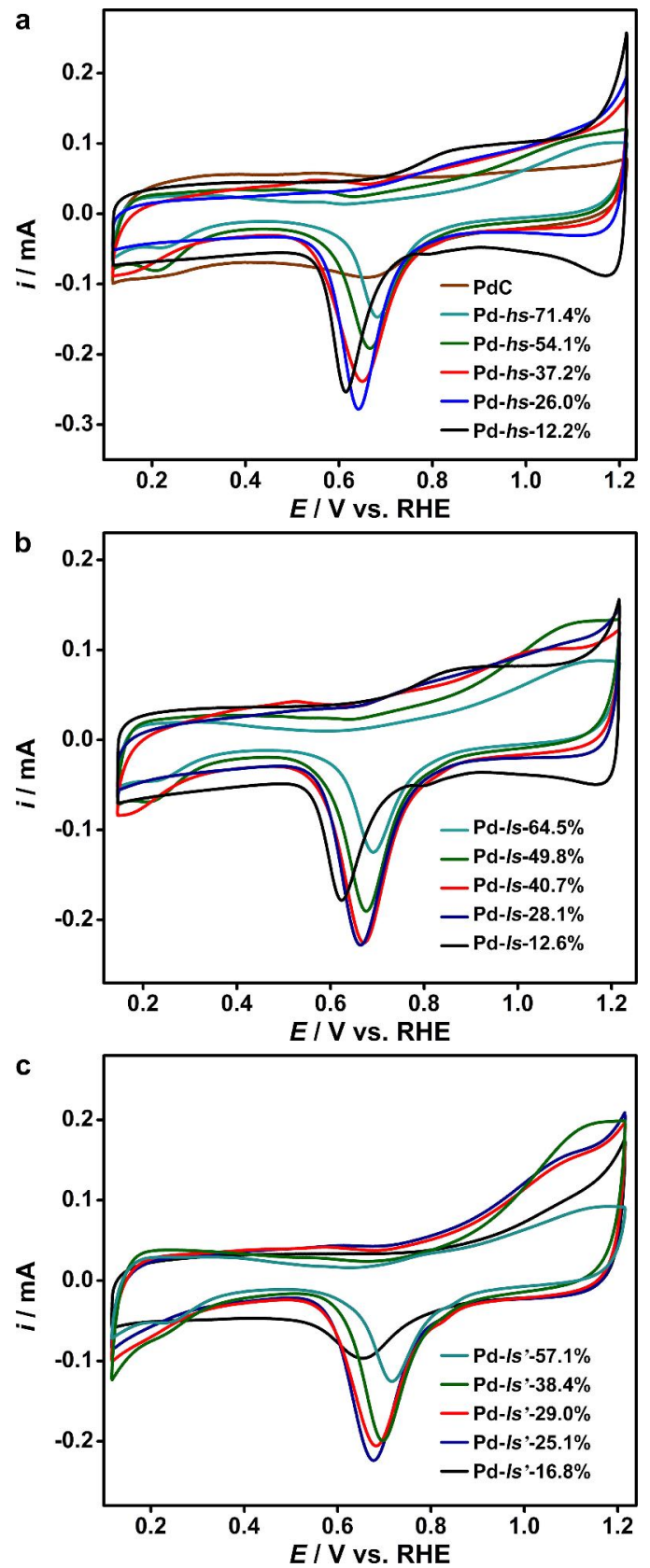

Figure S21. CV curves of the highly strained Au-Ag-Pd alloy nanowires (a: Pd- $h s-x)$ and the less strain Au-Ag-Pd alloy nanowires (b: Pd- $l s-x$; c: Pd- $l s^{\prime}-x$ ) in a potential range of $0.116-1.216 \mathrm{~V}_{\text {in N}} \mathrm{N}_{2}$-saturated $0.5 \mathrm{M} \mathrm{KOH}$ at $25^{\circ} \mathrm{C}$. Scan rate, $50 \mathrm{mV} \mathrm{s}^{-1}$. The cathodic peaks at $\sim 0.6-0.8 \mathrm{~V}$ correspond to the reduction of the PdO monolayer. The ECSAs of the catalysts were measured according to the coulombic charges associated with the reduction of the $\mathrm{PdO}$ monolayer assuming a reference value of $4.05 \mathrm{C} \mathrm{m}^{-2}$. The ECSA values of the catalysts are summarized in Table S4. 


\section{Discussion on $I_{f} / I_{b}$ ratio of the EGOR (Figure 4a):}

With a decreasing fraction of Pd in the highly stained Au-Ag-Pd alloy nanowires (Pd- $h s-x),{ }^{*} \mathrm{OH}$ adsorbs more strongly on the nanowires (Figure 3c), showing higher oxophilicity. The oxophilicity of the nanowires can be also reflected by the $I_{\mathrm{f}} / I_{\mathrm{b}}$ ratio in the $\mathrm{CV}$ curves of the alcohol oxidation reaction ( $\left(I_{\mathrm{f}}\right.$ :

forward oxidation peak current; $I_{\mathrm{b}}$ : backward oxidation peak current). ${ }^{7}$ The $I_{\mathrm{f}} / I_{\mathrm{b}}$ ratio increased from $\sim 1.0$ (Pd- $h s-71.4 \%$ and $\mathrm{Pd}-h s-54.1 \%)$ to $1.7(\mathrm{Pd}-h s-37.2 \%)$ and $2.4(\mathrm{Pd}-h s-26.0 \%)$ with a decreasing fraction of $\mathrm{Pd}$, confirming a continuous increase in the oxophilicity. An increase in the * OH adsorption energy (or the oxophilicity) leads to enhanced adsorption of EG and an increased population of surface *OH for the oxidation of EG and its intermediates, both contributing to the enhanced reaction kinetics. 

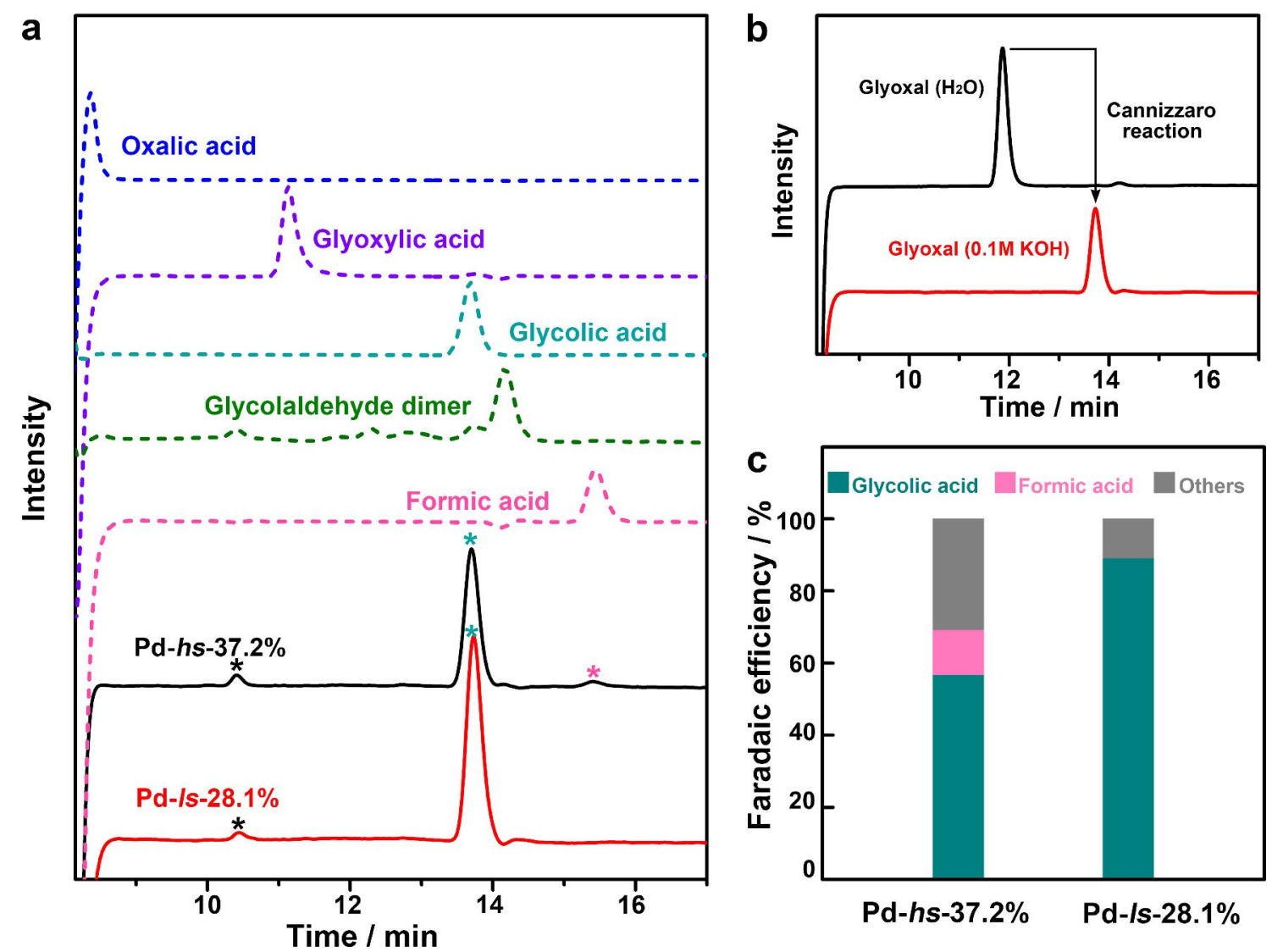

Figure S22. HPLC analysis of the EGOR products. (a) Solid lines: HPLC analysis of the electrolytes after the $i$ - $t$ tests at $0.8 \mathrm{~V}$. The quantity of the electricity was maintained at $50 \mathrm{C}$ during the electrocatalysis. Dotted lines: HPLC of the reference compounds possibly produced from the EGOR, including oxalic acid, glyoxylic acid, glycolic acid, glycolaldehyde, and formic acid. The reference compounds were purchased without purification and dissolved in $0.1 \mathrm{M} \mathrm{KOH}+0.5 \mathrm{M} \mathrm{EG}$ prior to the analysis. The black asterisks indicate the signals of impurities. The cyan and pink asterisks indicate the signals of glycolic acid and formic acid, respectively. (b) HPLC of glyoxal dissolved in water (black line) and an alkaline solution $(0.1 \mathrm{M} \mathrm{KOH}+0.5 \mathrm{M} \mathrm{EG}$, red line), showing the rapid conversion of glyoxal into glycolic acid under an alkaline condition through the Cannizzaro reaction. (c) Faradaic efficiencies of the products with the catalysts of $\mathrm{Pd}-h s-37.2 \%$ and $\mathrm{Pd}-l s-28.1 \%$. "Others" refer to other products of the glycol oxidation, mainly $\mathrm{CO}_{2}$, as a result of the complete oxidation of ethylene glycol. 
Discussion: Because the mobile phase is a strong acid in the HPLC analysis, all carboxylate products of the EGOR are converted into their carboxylic acid counterparts. Therefore, we use glyoxylic acid, oxalic acid, glycolic acid, and formic acid as reference compounds for the analysis (Figure S22a). Besides glycolaldehyde, another possible aldehyde product is glyoxal. However, glyoxal readily react with $\mathrm{KOH}$ in the electrolyte to form glycolic acid through the intramolecular disproportionation reaction (Cannizzaro reaction) without involving the electron transfer (Figure S22b). Therefore, glyoxal is not considered as a product of the alkaline ethylene glycol oxidation.

It can be inferred from Figure S22a that the main product of the EGOR with the catalysts of Pd-hs-37.2\% and Pd-ls-28.1\% is glycolic acid (cyan asterisks). Formic acid is another possible product (pink asterisks), but its concentration is much lower than glycolic acid. No obvious signals of glycolaldehyde, glyoxylic acid, or oxalic acid were observed in the HPLC analysis.

We further calculated the Faraday Efficiency of each product (Figure S22c). The EGOR with the highly strained Pd-hs-37.2\% catalyst affords a large amount of formic acid and $\mathrm{CO}_{2}$ with a Faraday efficiency of $43 \%$. In contrast, the EGOR with the less strained Pd-ls-28.1\% catalyst affords a much less formic acid (undetectable by HPLC) and $\mathrm{CO}_{2}$ with a Faraday efficiency of only $11 \%$. Because both formic acid and $\mathrm{CO}_{2}$ were produced only through the $\mathrm{C}-\mathrm{C}$ bond cleavage of the $\mathrm{EG}$ molecules, we conclude that the strong tensile strain in the $\mathrm{Pd}-h s-37.2 \%$ catalyst contributes to the $\mathrm{C}-\mathrm{C}$ bond cleavage and thus to the high overall efficiency of the EGOR. 

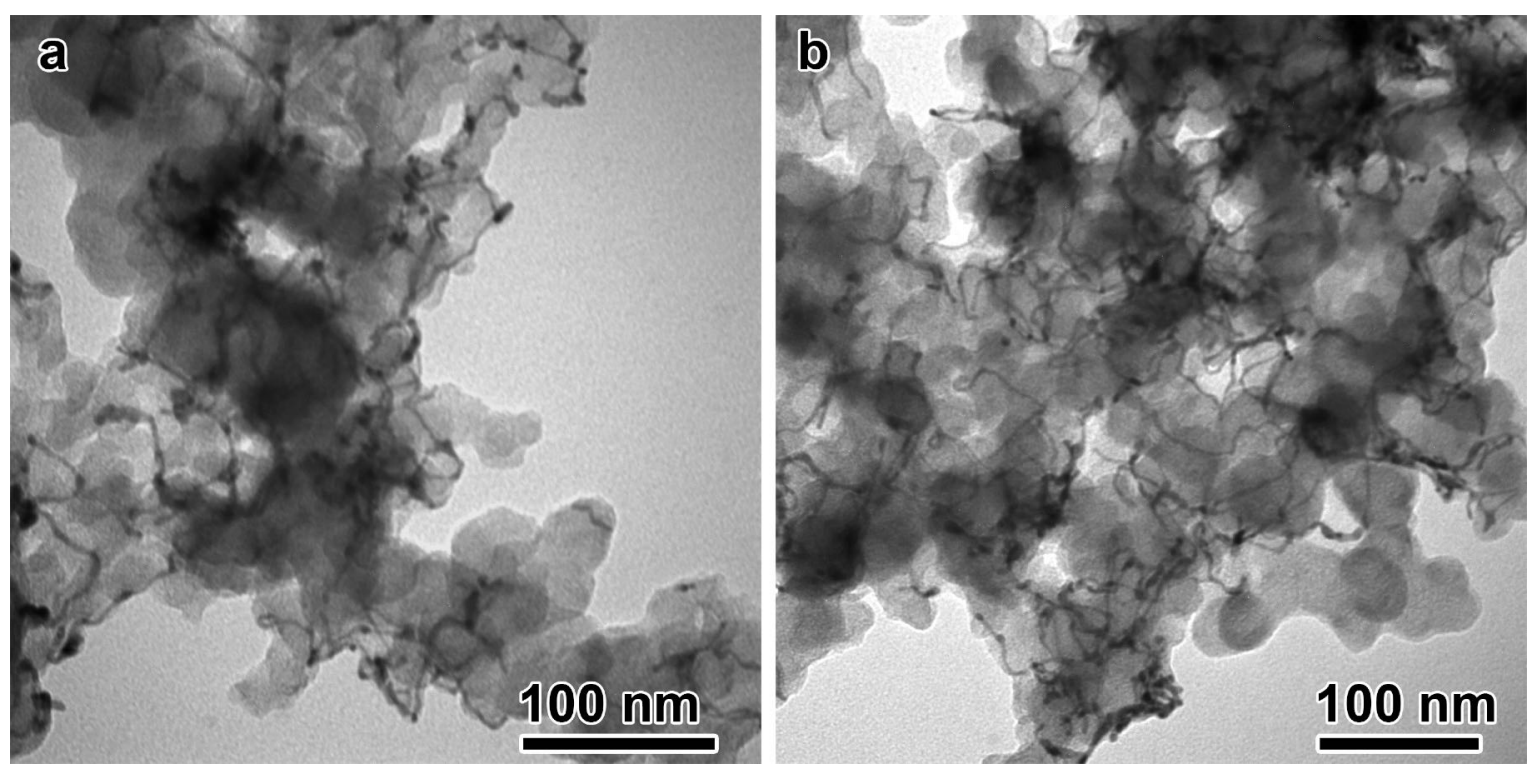

Figure S23. TEM images of the carbon-supported highly strained ultrathin Au-Ag-Pd alloy nanowires (Pd-hs-37.2\%) before (a) and after (b) the chronoamperometry measurements for $9 \mathrm{~h}$. The nanowires retain structural integrity and high dispersity on the carbon support during the electrocatalysis, which accounts for their long-term durability. 

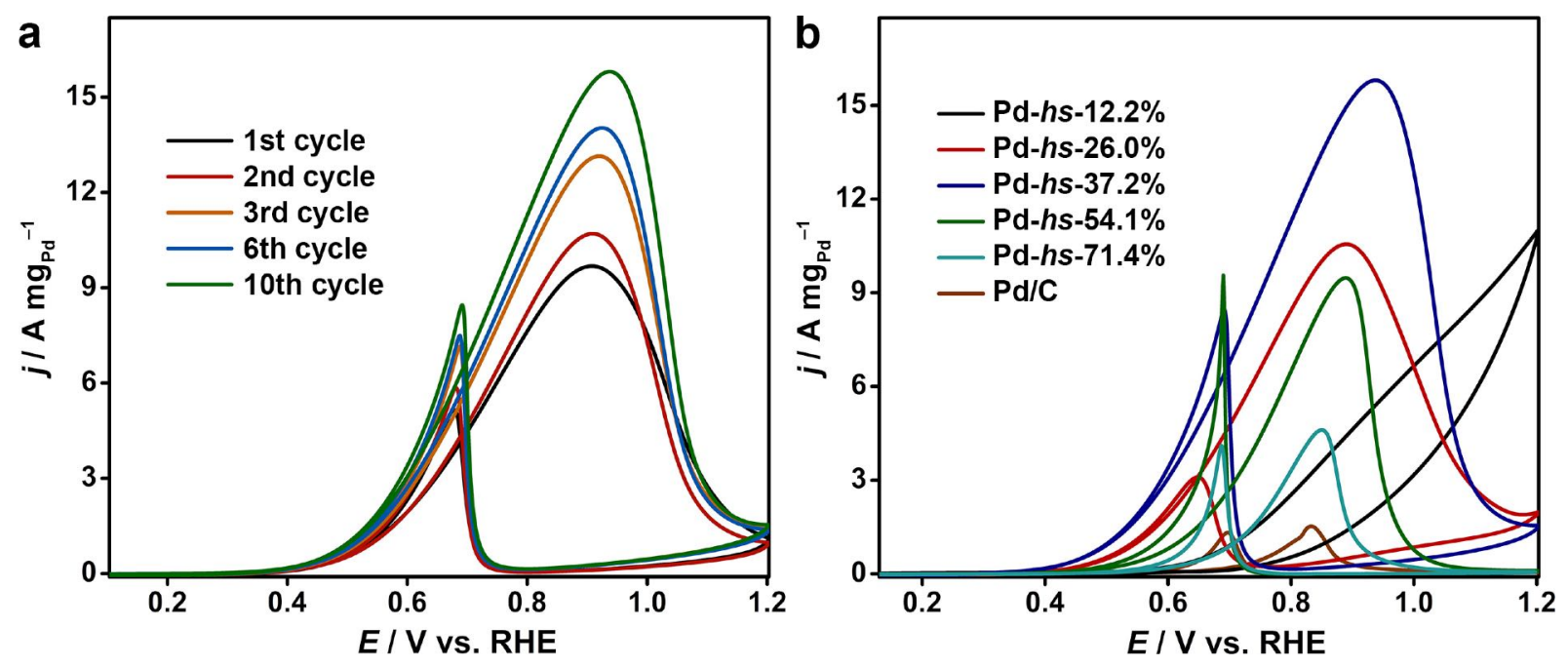

Figure S24. Electrocatalytic properties of the highly strained ultrathin Au-Ag-Pd alloy nanowires (Pd-hs$x$ ) after the durability $(i-t)$ test. The chronoamperometric $i$ - $t$ test was first carried out in $0.5 \mathrm{M} \mathrm{KOH}+0.5$ $\mathrm{M} \mathrm{EG}$ at $0.8 \mathrm{~V}$ for $9 \mathrm{~h}$. CV curves were then recorded with the electrodes in $0.5 \mathrm{M} \mathrm{KOH}+0.5 \mathrm{M}$ EG at a scan rate of $50 \mathrm{mV} \mathrm{s}^{-1}$. (a) Mass activity of the Pd- $h s-37.2 \%$ nanowires. The CV curves were scanned for 1-10 cycles, showing increasing activity which was stabilized at $15.8 \mathrm{~A} \mathrm{mg}_{\mathrm{Pd}^{-1}}$. (b) Mass activities of the Pd- $h s-x$ nanowires after the durability test. Discussion: (1) The nanowires retained a large portion of their initial activities after the long-term durability tests. Interestingly, the nanowires gradually recovered their catalytic activities by the $\mathrm{CV}$ activation. A reasonable explanation is that the carbonyl intermediates accumulated during the $i-t$ test were gradually oxidized at a high potential in the subsequent $\mathrm{CV}$ cycles, leading to a recovery of the catalytic activities. A surface reconstruction may also occur during the $i$ - $t$ test. The CV cycles may have recovered the surface structure of the catalysts, leading to increasing catalytic activities. (2) After the durability test, the nanowires showed a slight decrease in the ECSA and the mass activity. We assume that a leaching of Pd from the nanowires may have occurred during the long-term durability test. Nevertheless, the mass activity values of the nanowires after the durability test are close to those before the test (Figure 4a), which confirms the remarkable stability of the Au-Ag-Pd alloy nanowires in the catalysis. 

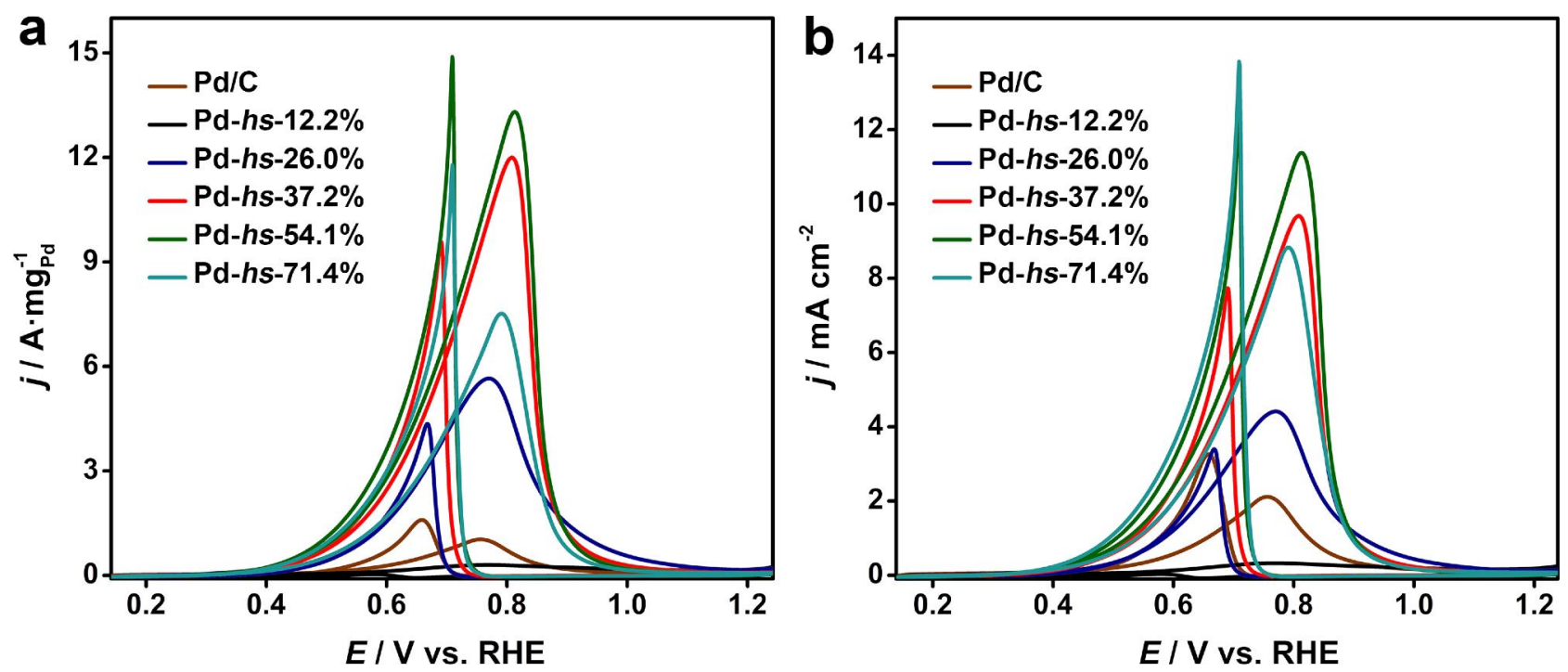

Figure S25. Electrocatalytic activities of the highly strained ultrathin Au-Ag-Pd alloy nanowires (Pd-hs$x$ ) in the ethanol oxidation reaction (EOR). The mass (a) and specific activities (b) are normalized to the mass and the ECSA of Pd, respectively. All measurements were carried out in $\mathrm{N}_{2}$-saturated $1 \mathrm{M} \mathrm{KOH}+$ $1 \mathrm{M}$ ethanol at a scan rate of $50 \mathrm{mV} \mathrm{s}^{-1}$. The Pd-hs-54.1\% nanowires exhibit the highest mass (13.6 A $\mathrm{mg}_{\mathrm{Pd}^{-1}}{ }^{-1}$ and specific activity $\left(11.8 \mathrm{~mA} \mathrm{~cm}{ }^{-2}\right)$. 

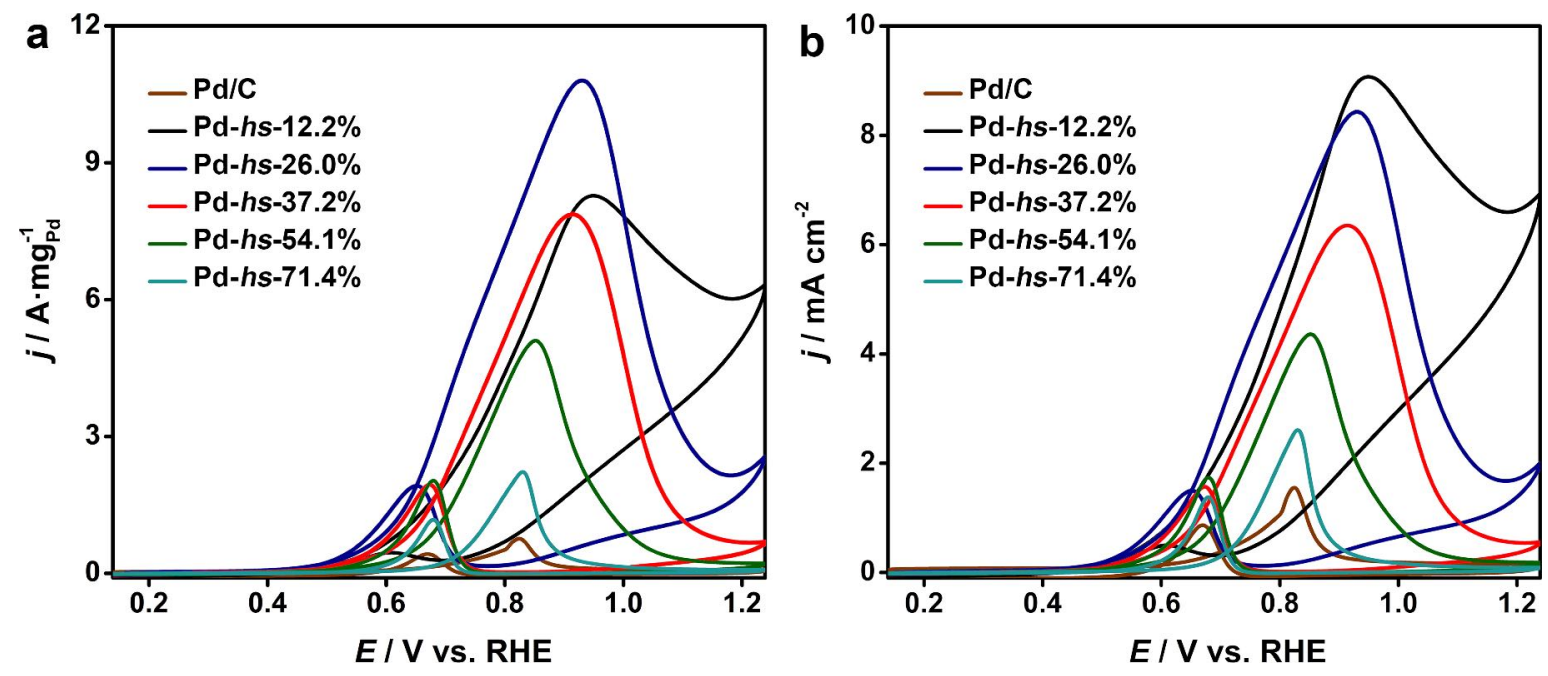

Figure S26. Electrocatalytic activities of the ultrathin Au-Ag-Pd alloy nanowires (Pd- $h s-x)$ in the glycerol oxidation reaction (GOR). The mass (a) and specific activities (b) are normalized to the mass and the ECSA of Pd, respectively. All measurements were carried out in $\mathrm{N}_{2}$-saturated $1 \mathrm{M} \mathrm{KOH}+0.1 \mathrm{M}$ glycerol at a scan rate of $50 \mathrm{mV} \mathrm{s}^{-1}$. The Pd- $h s-26.0 \%$ nanowires exhibit the highest mass activity of $11.1 \mathrm{~A} \mathrm{mg}_{\mathrm{Pd}}{ }^{-1}$. 

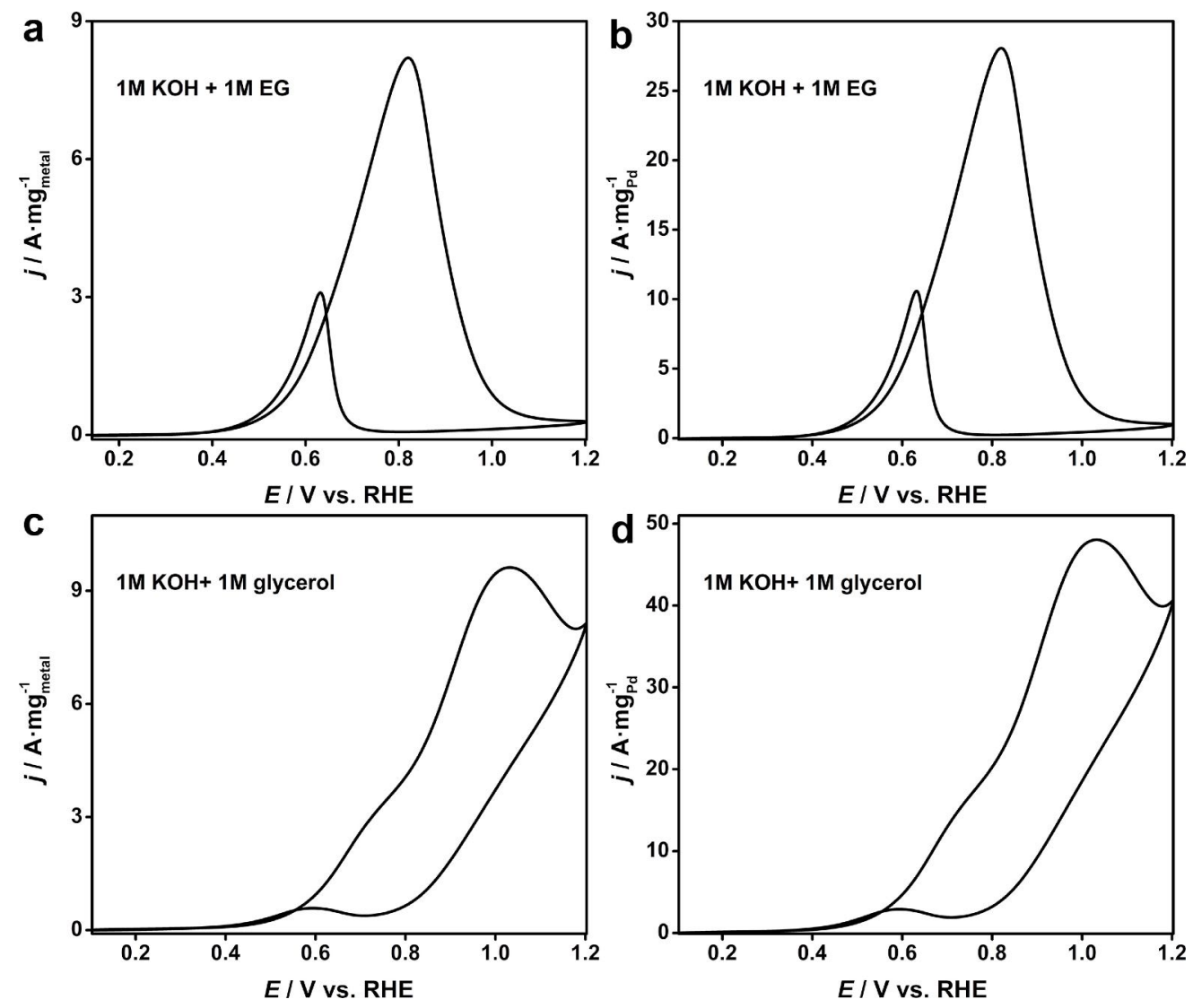

Figure S27. Electrocatalytic activities of the Pd- $h s-37.2 \%$ in the EGOR (a, b) and Pd- $h s-26.0 \%$ in the GOR (c, d). The mass activities were normalized to the mass of all metals and the mass of Pd, respectively. The measurements were carried out in $\mathrm{N}_{2}$-saturated $1 \mathrm{M} \mathrm{KOH}+1 \mathrm{M}$ ethylene glycol (or glycerol) at a scan rate of $50 \mathrm{mV} \mathrm{s}^{-1}$. The Pd- $h s-37.2 \%$ nanowires exhibit a mass activity of $27.5 \mathrm{~A} \mathrm{mg}_{\mathrm{Pd}}^{-1}$ in the EGOR. The Pd-hs-26.0\% nanowires exhibit a mass activity of $48.0 \mathrm{~A} \mathrm{mg}_{\mathrm{Pd}}{ }^{-1}$ in the GOR. All activities were measured at their peak potentials. 
Table S1. Molar composition of the highly strained Au-Ag-Pd alloy nanowires (Pd-hs-x).

\begin{tabular}{cccc}
\hline Catalysts & $\mathrm{Au}: \mathrm{Ag}: \mathrm{Pd}$ & $\mathrm{Au} / \mathrm{Ag}$ & $\mathrm{Pd} /(\mathrm{Ag}+\mathrm{Au}+\mathrm{Pd})$ \\
\hline Pd-hs-12.2\% & $0.42: 0.45: 0.12$ & 0.93 & 0.122 \\
Pd- $h s-26.0 \%$ & $0.34: 0.40: 0.26$ & 0.86 & 0.260 \\
Pd- $h s-37.2 \%$ & $0.32: 0.31: 0.37$ & 1.00 & 0.372 \\
Pd- $h s-54.1 \%$ & $0.23: 0.23: 0.54$ & 1.03 & 0.541 \\
Pd- $h s-71.4 \%$ & $0.15: 0.14: 0.71$ & 1.10 & 0.714 \\
\hline
\end{tabular}


Table S2. Molar composition of the less strained Au-Ag-Pd alloy nanowires (Pd-ls-x).

\begin{tabular}{cccc}
\hline Catalysts & $\mathrm{Au}: \mathrm{Ag}: \mathrm{Pd}$ & $\mathrm{Au} / \mathrm{Ag}$ & $\mathrm{Pd} /(\mathrm{Ag}+\mathrm{Au}+\mathrm{Pd})$ \\
\hline Pd- $l s-12.6 \%$ & $0.47: 0.40: 0.13$ & 1.16 & 0.126 \\
Pd- $l s-28.1 \%$ & $0.39: 0.33: 0.28$ & 1.19 & 0.281 \\
Pd- $l s-40.7 \%$ & $0.32: 0.27: 0.41$ & 1.18 & 0.407 \\
Pd- $l s-49.8 \%$ & $0.27: 0.23: 0.50$ & 1.16 & 0.498 \\
Pd- $l s-64.5 \%$ & $0.20: 0.16: 0.64$ & 1.25 & 0.645 \\
\hline
\end{tabular}


Table S3. Molar composition of the less strained Au-Ag-Pd alloy nanowires (Pd-ls'-x).

\begin{tabular}{cccc}
\hline Catalysts & $\mathrm{Au}: \mathrm{Ag}: \mathrm{Pd}$ & $\mathrm{Au} / \mathrm{Ag}$ & $\mathrm{Pd} /(\mathrm{Ag}+\mathrm{Au}+\mathrm{Pd})$ \\
\hline Pd- $l s^{\prime}-16.8 \%$ & $0.41: 0.42: 0.17$ & 0.98 & 0.168 \\
Pd- $l s^{\prime}-25.1 \%$ & $0.34: 0.42: 0.25$ & 0.83 & 0.251 \\
Pd- $l s^{\prime}-29.0 \%$ & $0.32: 0.39: 0.29$ & 0.82 & 0.290 \\
Pd- $l s^{\prime}-38.4 \%$ & $0.30: 0.31: 0.38$ & 0.97 & 0.384 \\
Pd- $l s^{\prime}-57.1 \%$ & $0.20: 0.23: 0.57$ & 0.90 & 0.571 \\
\hline
\end{tabular}


Table S4. ECSAs of the Au-Ag-Pd alloy nanowires (Pd- $h s-x, \mathrm{Pd}-l s-x$, and $\left.\mathrm{Pd}-l s^{\prime}-x\right)$ and the $\mathrm{Pd} / \mathrm{C}$.

\begin{tabular}{cc}
\hline Catalysts & ECSA $/ \mathrm{m}^{2} \mathrm{gd}^{-1}$ \\
\hline Pd- $h s^{-12.2 \%}$ & 88 \\
Pd- $h s^{-26.0 \%}$ & 129 \\
Pd- $h s^{-37.2 \%}$ & 126 \\
Pd- $h s^{-54.1 \%}$ & 115 \\
Pd- $h s^{-}-71.4 \%$ & 84 \\
Pd- $l s^{-}-12.6 \%$ & 69 \\
Pd- $l s^{-}-28.1 \%$ & 124 \\
Pd- $l s^{-}-40.7 \%$ & 132 \\
Pd- $l s^{-}-49.8 \%$ & 115 \\
Pd- $l s^{-}-64.5 \%$ & 73 \\
Pd- $l s^{\prime}-16.8 \%$ & 53 \\
Pd- $l s^{\prime}-25.1 \%$ & 132 \\
Pd- $l s^{\prime}-29.0 \%$ & 129 \\
Pd- $l s^{\prime}-38.4 \%$ & 120 \\
Pd- $l s^{\prime}-57.1 \%$ & 79 \\
Commercial Pd/C & 47 \\
\hline
\end{tabular}


Table S5. A comparison of the EGOR catalytic activities of the typical Pd, Pt-based catalysts reported in the literature.

\begin{tabular}{|c|c|c|c|c|}
\hline Electrocatalysts & Reaction Medium & $\begin{array}{l}\text { Mass Activity } \\
\quad\left(\mathrm{A} \mathrm{mg}^{-1}\right)\end{array}$ & $\begin{array}{c}\text { Specific } \\
\text { Activity } \\
\left.(\mathrm{mA} \mathrm{cm})^{-2}\right)\end{array}$ & Reference \\
\hline \multirow{2}{*}{$\begin{array}{l}\text { Highly strained } \\
\text { ultrathin Au-Ag-Pd } \\
\text { alloy nanowires }\end{array}$} & $\begin{array}{l}0.5 \mathrm{M} \mathrm{KOH} \mathrm{+} \\
0.5 \mathrm{M} \mathrm{EG}\end{array}$ & 18.2 & 14.5 & \multirow{2}{*}{ This work } \\
\hline & $\begin{array}{c}1 \mathrm{M} \mathrm{KOH} \mathrm{+} \\
1 \mathrm{M} \mathrm{EG}\end{array}$ & 27.5 & - & \\
\hline $\begin{array}{c}\text { Au-island-covered } \mathbf{P d} \\
\text { nanotubes }\end{array}$ & $\begin{array}{c}0.5 \mathrm{M} \mathrm{KOH}+ \\
0.5 \mathrm{M} \mathrm{EG}\end{array}$ & $\sim 7.53$ & - & 8 \\
\hline $\begin{array}{c}\text { PdAuNi } \\
\text { nanosponges }\end{array}$ & $\begin{array}{c}0.5 \mathrm{M} \mathrm{KOH}+ \\
0.5 \mathrm{M} \mathrm{EG}\end{array}$ & 6.36 & - & 9 \\
\hline PdAg nanosheets & $\begin{array}{l}0.5 \mathrm{M} \mathrm{KOH}+ \\
1 \mathrm{MEG}\end{array}$ & 7.01 & 14.1 & 10 \\
\hline Pd-Ru nanocages & $\begin{array}{c}1 \mathrm{M} \mathrm{KOH}+ \\
1 \mathrm{MEG}\end{array}$ & 5.54 & & 11 \\
\hline PdAuAg nanocages & $\begin{array}{c}1 \mathrm{M} \mathrm{KOH}+ \\
1 \mathrm{MEG}\end{array}$ & $\begin{array}{c}7.58 \\
\mathrm{~A} \mathrm{mg}_{\text {metal }}^{-1}\end{array}$ & - & 12 \\
\hline Pd-WO $\mathrm{WO}_{2.75}$ nanobelts & $\begin{array}{c}1 \mathrm{M} \mathrm{KOH}+ \\
1 \mathrm{MEG}\end{array}$ & 4.58 & - & 13 \\
\hline PdAu nanoflowers/NG & $\begin{array}{c}1 \mathrm{M} \mathrm{KOH}+ \\
1 \mathrm{MEG}\end{array}$ & 12.8 & - & 14 \\
\hline Ag-rich PdAg & $\begin{array}{c}1 \mathrm{M} \mathrm{NaOH}+ \\
1 \mathrm{MEG}\end{array}$ & 7.93 & 7.8 & 15 \\
\hline $\begin{array}{c}\text { PdAg } \\
\text { nanoparticles }\end{array}$ & $\begin{array}{c}1 \mathrm{M} \mathrm{KOH}+ \\
1 \mathrm{MEG}\end{array}$ & 5.33 & 21.4 & 16 \\
\hline PdCu nanosheets & $\begin{array}{c}1 \mathrm{M} \mathrm{KOH}+ \\
1 \mathrm{MEG}\end{array}$ & 4.71 & 13.7 & 17 \\
\hline $\mathbf{P d P b}$ nanocubes & $\begin{array}{c}1 \mathrm{M} \mathrm{KOH}+ \\
1 \mathrm{MEG}\end{array}$ & 4.06 & 16.8 & 18 \\
\hline $\mathbf{P d} / \mathrm{MGF}$ & $\begin{array}{c}1 \mathrm{M} \mathrm{KOH}+ \\
1 \mathrm{M} \mathrm{EG}\end{array}$ & 4.06 & - & 19 \\
\hline
\end{tabular}




\begin{tabular}{|c|c|c|c|c|}
\hline PdCuTe nanowires & $\begin{array}{c}1 \mathrm{M} \mathrm{KOH}+ \\
1 \mathrm{MEG}\end{array}$ & 3.87 & 7.9 & 20 \\
\hline $\begin{array}{l}\text { Concave Pd-Ru } \\
\text { nanocubes }\end{array}$ & $\begin{array}{c}1 \mathrm{M} \mathrm{KOH}+ \\
1 \mathrm{MEG}\end{array}$ & 3.35 & - & 21 \\
\hline $\mathbf{P d} / \mathrm{RuO}_{2}-\mathrm{SnO}_{2} / \mathrm{C}$ & $\begin{array}{l}1 \mathrm{M} \mathrm{KOH}+ \\
1 \mathrm{M} \mathrm{EG} .\end{array}$ & 3.03 & 4.4 & 22 \\
\hline $\begin{array}{c}\text { Ultrathin } \\
\text { PtPdNi nanosheets }\end{array}$ & $\begin{array}{l}0.5 \mathrm{M} \mathrm{KOH}+ \\
0.5 \mathrm{M} \mathrm{EG}\end{array}$ & $\sim 11$ & - & 23 \\
\hline $\begin{array}{c}\text { Bimetallic PdPt } \\
\text { nanowires }\end{array}$ & $\begin{array}{l}0.5 \mathrm{M} \mathrm{KOH}+ \\
0.5 \mathrm{M} \mathrm{EG}\end{array}$ & 3.38 & - & 24 \\
\hline PtPd nanodendrites & $\begin{array}{c}1 \mathrm{M} \mathrm{KOH}+ \\
0.5 \mathrm{M} \mathrm{EG}\end{array}$ & 1.64 & 5.2 & 25 \\
\hline UsPtCu@C & $\begin{array}{l}0.5 \mathrm{M} \mathrm{KOH}+ \\
1 \mathrm{MEG}\end{array}$ & 8.5 & 3.7 & 26 \\
\hline $\begin{array}{c}\text { 3D PtPb nanowire } \\
\text { networks }\end{array}$ & $\begin{array}{c}0.5 \mathrm{M} \mathrm{NaOH}+ \\
0.5 \mathrm{M} \mathrm{EG}\end{array}$ & 13.5 & 5.7 & 27 \\
\hline $\begin{array}{l}\text { Dendritic Pt-Co } \\
\text { nanocubes }\end{array}$ & $\begin{array}{l}0.5 \mathrm{M} \mathrm{KOH}+ \\
0.5 \mathrm{M} \mathrm{EG}\end{array}$ & $\sim 2.6$ & 7.8 & 28 \\
\hline $\begin{array}{c}\text { AuPt@Pt core-shell } \\
\text { nanocrystals }\end{array}$ & $\begin{array}{l}0.5 \mathrm{M} \mathrm{KOH}+ \\
0.5 \mathrm{M} \mathrm{EG}\end{array}$ & 1.85 & - & 29 \\
\hline $\begin{array}{l}\text { PtCuCo hollow } \\
\text { nanospheres }\end{array}$ & $\begin{array}{c}0.5 \mathrm{M} \mathrm{NaOH}+ \\
0.5 \mathrm{M} \mathrm{EG}\end{array}$ & 1.8 & - & 30 \\
\hline PtRh nanocubes & $\begin{array}{l}1 \mathrm{M} \mathrm{KOH}+ \\
1 \mathrm{M} \mathrm{EG}\end{array}$ & 5.13 & 11.6 & 31 \\
\hline $\begin{array}{c}\mathrm{CeO}_{2} \cdot x \mathrm{H}_{2} \mathrm{O} / \mathbf{P t} / \text { carbon } \\
\text { nanotubes }\end{array}$ & $\begin{array}{l}1 \mathrm{M} \mathrm{KOH}+ \\
1 \mathrm{M} \mathrm{EG}\end{array}$ & 3.25 & - & 32 \\
\hline
\end{tabular}

Notes: The mass activities were calculated by normalizing the activity to the mass of Pd (for Pd-based catalysts), Pt (for Pt-based catalysts), or Pt + Pd (for Pt-Pd based catalysts), unless otherwise stated. The highly strained ultrathin Au-Ag-Pd alloy nanowires obtained in this work demonstrate mass activities of 18.2 $\mathrm{A} \mathrm{mg}_{\mathrm{Pd}^{-1}}$ in $0.5 \mathrm{M} \mathrm{KOH}+0.5 \mathrm{M} \mathrm{EG}$ and $27.5 \mathrm{~A} \mathrm{mg}_{\mathrm{Pd}^{-1}}$ in $1 \mathrm{M} \mathrm{KOH}+1 \mathrm{M} \mathrm{EG}$, which are recordhigh values among all activities reported in the literature under the similar conditions. 
Table S6. A comparison of the GOR catalytic activities of the typical Pd, Pt-based catalysts reported in the literature.

\begin{tabular}{|c|c|c|c|c|}
\hline Electro-catalysts & Reaction Medium & $\begin{array}{l}\text { Mass Activity } \\
\quad\left(\mathrm{A} \mathrm{mg}^{-1}\right)\end{array}$ & $\begin{array}{c}\text { Specific } \\
\text { Activity } \\
\left.(\mathrm{mA} \mathrm{cm})^{-2}\right)\end{array}$ & Reference \\
\hline \multirow{2}{*}{$\begin{array}{c}\text { Ultrathin highly } \\
\text { strained Au-Ag-Pd alloy } \\
\text { nanowires }\end{array}$} & $\begin{array}{l}1 \mathrm{M} \mathrm{KOH} \mathrm{+} \\
0.1 \mathrm{M} \text { glycerol }\end{array}$ & 11.1 & 8.6 & \multirow{2}{*}{ This work } \\
\hline & $\begin{array}{l}1 \mathrm{M} \mathrm{KOH} \mathrm{+} \\
1 \mathrm{M} \text { glycerol }\end{array}$ & $\begin{array}{l}\text { 48.0 } \mathrm{A} \mathrm{mg}_{\mathrm{Pd}^{-1}}{ }^{-1} \\
\text { 9.6A mgetal }\end{array}$ & - & \\
\hline $\begin{array}{c}\text { PdAg supported on carbon } \\
\text { nanotubes }\end{array}$ & $\begin{array}{c}1 \mathrm{M} \mathrm{KOH} \mathrm{+} \\
0.1 \mathrm{M} \mathrm{glycerol}\end{array}$ & 8.53 & - & 33 \\
\hline $\begin{array}{c}\text { PdAuNi } \\
\text { nanosponges }\end{array}$ & $\begin{array}{c}1 \mathrm{M} \mathrm{KOH} \mathrm{+} \\
0.1 \mathrm{M} \mathrm{glycerol}\end{array}$ & 3.3 & - & 9 \\
\hline $\begin{array}{l}\text { Au-island-covered } \\
\text { Pd nanotubes }\end{array}$ & $\begin{array}{c}1 \mathrm{M} \mathrm{KOH} \mathrm{+} \\
0.1 \mathrm{M} \text { glycerol }\end{array}$ & $\sim 3.0$ & - & 8 \\
\hline $\mathbf{P d} \mathbf{d}_{x} \mathrm{Bi}$ & $\begin{array}{c}1 \mathrm{M} \mathrm{KOH} \mathrm{+} \\
0.1 \mathrm{M} \text { glycerol }\end{array}$ & $\sim 0.8$ & - & 34 \\
\hline $\begin{array}{c}\text { AuPd@Pd core-shell } \\
\text { nanocrystals }\end{array}$ & $\begin{array}{l}0.5 \mathrm{M} \mathrm{KOH} \mathrm{+} \\
0.5 \mathrm{M} \mathrm{glycerol}\end{array}$ & 1.1 & - & 35 \\
\hline Porous coral-like $\mathbf{P d A g}$ & $\begin{array}{c}1 \mathrm{M} \mathrm{KOH} \mathrm{+} \\
0.5 \mathrm{M} \mathrm{glycerol}\end{array}$ & 1.60 & 6.07 & 36 \\
\hline PdAuAg nanocages & $\begin{array}{l}1 \mathrm{M} \mathrm{KOH}+ \\
1 \mathrm{M} \text { glycerol }\end{array}$ & $\begin{array}{c}5.68 \\
\mathrm{~A} \mathrm{mg}_{\text {metal }}^{-1}\end{array}$ & - & 12 \\
\hline PdAu nanoflowers/NG & $\begin{array}{l}1 \mathrm{M} \mathrm{KOH}+ \\
1 \mathrm{M} \text { glycerol }\end{array}$ & 8.7 & - & 14 \\
\hline Pd-WO $\mathrm{WO}_{2.75}$ nanobelts & $\begin{array}{l}1 \mathrm{M} \mathrm{KOH} \mathrm{+} \\
1 \mathrm{M} \mathrm{glycerol}\end{array}$ & 4.9 & - & 13 \\
\hline PdAg nanoparticles & $\begin{array}{l}1 \mathrm{M} \mathrm{KOH} \mathrm{+} \\
1 \mathrm{M} \mathrm{glycerol}\end{array}$ & 3.51 & 14.1 & 16 \\
\hline Pd/MGF & $\begin{array}{l}1 \mathrm{M} \mathrm{KOH} \mathrm{+} \\
1 \mathrm{M} \text { glycerol }\end{array}$ & 2.72 & - & 19 \\
\hline
\end{tabular}




\begin{tabular}{|c|c|c|c|c|}
\hline $\begin{array}{l}\text { Binary } \mathbf{P d P b} \text { alloy } \\
\text { nanocubes }\end{array}$ & $\begin{array}{l}1 \mathrm{M} \mathrm{KOH} \mathrm{+} \\
1 \mathrm{M} \mathrm{glycerol}\end{array}$ & 2.22 & 9.2 & 18 \\
\hline $\begin{array}{c}\text { PdBi } \\
\text { nanosheet assemblies }\end{array}$ & $\begin{array}{l}1 \mathrm{M} \mathrm{KOH}+ \\
1 \mathrm{M} \text { glycerol }\end{array}$ & 3.04 & 7.9 & 37 \\
\hline $\begin{array}{c}\text { Pd-Cu-Pt trimetallic twin } \\
\text { icosahedrons }\end{array}$ & $\begin{array}{l}1 \mathrm{M} \mathrm{KOH}+ \\
1 \mathrm{M} \text { glycerol }\end{array}$ & 9.7 & & 38 \\
\hline $\begin{array}{l}\text { Ultrathin PtPdNi } \\
\text { nanosheets }\end{array}$ & $\begin{array}{c}1 \mathrm{M} \mathrm{KOH} \mathrm{+} \\
0.1 \mathrm{M} \text { glycerol }\end{array}$ & $\sim 6$ & - & 23 \\
\hline $\begin{array}{l}\text { CuPdPt ultrathin } \\
\text { nanowire networks }\end{array}$ & $\begin{array}{c}1 \mathrm{M} \mathrm{KOH} \mathrm{+} \\
0.1 \mathrm{M} \text { glycerol }\end{array}$ & 2.16 & - & 39 \\
\hline Bimetallic PdPt nanowires & $\begin{array}{c}1 \mathrm{M} \mathrm{KOH} \mathrm{+} \\
0.1 \mathrm{M} \mathrm{glycerol}\end{array}$ & 1.8 & - & 24 \\
\hline PtAg nanotubes & $\begin{array}{l}0.5 \mathrm{M} \mathrm{NaOH}+ \\
0.5 \mathrm{M} \text { glycerol }\end{array}$ & $\sim 1.6$ & - & 40 \\
\hline PtPd & $\begin{array}{l}0.5 \mathrm{M} \mathrm{KOH} \mathrm{+} \\
0.5 \mathrm{M} \text { glycerol }\end{array}$ & 1.53 & 2.74 & 41 \\
\hline Dendritic Pt-Co nanocubes & $\begin{array}{c}1 \mathrm{M} \mathrm{KOH} \mathrm{+} \\
0.1 \mathrm{M} \mathrm{glycerol}\end{array}$ & $\sim 1.1$ & 3.53 & 28 \\
\hline $\begin{array}{c}\mathrm{CeO}_{2} \cdot x \mathrm{H}_{2} \mathrm{O} / \mathbf{P t} / \text { carbon } \\
\text { nanotubes }\end{array}$ & $\begin{array}{l}1 \mathrm{M} \mathrm{KOH} \mathrm{+} \\
1 \mathrm{M} \text { glycerol }\end{array}$ & 1.88 & - & 32 \\
\hline
\end{tabular}

Notes: The mass activities were calculated by normalizing the activity to the mass of Pd (for Pd-based catalysts), Pt (for Pt-based catalysts), or Pt + Pd (for Pt-Pd based catalysts), unless otherwise stated. The highly strained ultrathin Au-Ag-Pd alloy nanowires obtained in this work demonstrate a mass activity of $11.1 \mathrm{~A} \mathrm{mg}_{\mathrm{Pd}^{-1}}{ }^{-1}$ in $1 \mathrm{M} \mathrm{KOH}+0.1 \mathrm{M}$ glycerol, which is a record-high value among all activities reported in the literature under the similar conditions. The ultrathin Au-Ag-Pd alloy nanowires also showed a mass activity of $9.6 \mathrm{~A} \mathrm{mg}_{\text {metal }}^{-1}$ in $1 \mathrm{M} \mathrm{KOH}+1 \mathrm{M}$ glycerol when the activity was normalized to the total mass of all metals. Although this activity was also high, it cannot be directly compared with the values reported in the literature under similar reaction conditions, because the GOR peaks appeared at different potentials. 
Table S7. A comparison of the EOR catalytic activities of the typical Pd, Pt-based catalysts reported in the literature.

\begin{tabular}{|c|c|c|c|c|}
\hline Electro-catalysts & Reaction Medium & $\begin{array}{l}\text { Mass Activity } \\
\quad\left(\mathrm{A} \mathrm{mg}^{-1}\right)\end{array}$ & $\begin{array}{c}\text { Specific } \\
\text { Activity } \\
\left.(\mathrm{mA} \mathrm{cm})^{-2}\right)\end{array}$ & Reference \\
\hline $\begin{array}{c}\text { Ultrathin highly } \\
\text { strained Au-Ag-Pd alloy } \\
\text { nanowires }\end{array}$ & $\begin{array}{l}\text { 1.0 M KOH + } \\
1.0 \mathrm{M} \text { ethanol }\end{array}$ & 13.6 & 11.8 & This work \\
\hline $\mathbf{P d}_{3} \mathrm{~Pb} \mathrm{NCs}$ & $\begin{array}{l}1.0 \mathrm{M} \mathrm{KOH}+ \\
1.0 \mathrm{M} \text { ethanol }\end{array}$ & 2.46 & 10.4 & 42 \\
\hline c-Pd-Ni-P@a-Pd-Ni-P NPs & $\begin{array}{l}1.0 \mathrm{M} \mathrm{KOH}+ \\
1.0 \mathrm{M} \text { ethanol }\end{array}$ & 3.05 & & 43 \\
\hline CoP/RGO-Pd & $\begin{array}{l}1.0 \mathrm{M} \mathrm{KOH} \mathrm{+} \\
1.0 \mathrm{M} \text { ethanol }\end{array}$ & 4.60 & 10 & 44 \\
\hline $\mathrm{Au} @ \mathbf{P d A u C u} \mathrm{MNSs}$ & $\begin{array}{l}1.0 \mathrm{M} \mathrm{KOH} \mathrm{+} \\
1.0 \mathrm{M} \text { ethanol }\end{array}$ & 3.99 & - & 45 \\
\hline PdAg NWs & $\begin{array}{l}1.0 \mathrm{M} \mathrm{KOH}+ \\
1.0 \mathrm{M} \text { ethanol }\end{array}$ & 2.84 & - & 46 \\
\hline PdAgCu MNSs & $\begin{array}{l}1.0 \mathrm{M} \mathrm{KOH} \mathrm{+} \\
1.0 \mathrm{M} \text { ethanol }\end{array}$ & 4.64 & - & 47 \\
\hline $\mathbf{P d} / \mathrm{Ag}-\mathrm{BP}$ & $\begin{array}{l}1.0 \mathrm{M} \mathrm{NaOH}+ \\
1.0 \mathrm{M} \text { ethanol }\end{array}$ & 6.0 & - & 48 \\
\hline $\mathrm{Bi}(\mathrm{OH})_{3} / \mathbf{P d B i}$ & $\begin{array}{l}1.0 \mathrm{M} \mathrm{NaOH}+ \\
1.0 \mathrm{M} \text { ethanol }\end{array}$ & 5.3 & - & 49 \\
\hline PdAg NDs & $\begin{array}{l}1.0 \mathrm{M} \mathrm{KOH}+ \\
1.0 \mathrm{M} \text { ethanol }\end{array}$ & 2.63 & 3.7 & 50 \\
\hline $\mathbf{P} \mathbf{d}_{4} \mathrm{Au}_{1}-\mathrm{P} / \mathrm{CNT}$ & $\begin{array}{l}1.0 \mathrm{M} \mathrm{KOH}+ \\
1.0 \mathrm{M} \text { ethanol }\end{array}$ & 2.29 & - & 51 \\
\hline 4H/fcc Au@Pd NRs & $\begin{array}{l}1.0 \mathrm{M} \mathrm{KOH}+ \\
1.0 \mathrm{M} \text { ethanol }\end{array}$ & 2.92 & - & 52 \\
\hline $\mathbf{P d A g} / \mathrm{rGO}$ & $\begin{array}{l}1.0 \mathrm{M} \mathrm{KOH}+ \\
1.0 \mathrm{M} \text { ethanol }\end{array}$ & 3.13 & 1.2 & 53 \\
\hline $\mathbf{P d} / \mathrm{Ni}(\mathrm{OH})_{2} / \mathrm{rGO}$ & $\begin{array}{l}1.0 \mathrm{M} \mathrm{KOH} \mathrm{+} \\
1.0 \mathrm{M} \text { ethanol }\end{array}$ & 1.54 & 4.1 & 54 \\
\hline
\end{tabular}




\begin{tabular}{|c|c|c|c|c|}
\hline PdNi-P & $\begin{array}{l}\text { 1.0 M KOH + } \\
1.0 \mathrm{M} \text { ethanol }\end{array}$ & 4.95 & - & 55 \\
\hline $\mathbf{P d N i}-\mathrm{HNCs}-\mathrm{R} / \mathrm{C}$ & $\begin{array}{l}\text { 1.0 M KOH + } \\
1.0 \mathrm{M} \text { ethanol }\end{array}$ & 1.2 & 4.12 & 56 \\
\hline PdCu NWs & $\begin{array}{l}1.0 \mathrm{M} \mathrm{KOH} \mathrm{+} \\
1.0 \mathrm{M} \text { ethanol }\end{array}$ & 3.47 & - & 57 \\
\hline PdNi HNS & $\begin{array}{c}1.0 \mathrm{M} \mathrm{NaOH}+ \\
1.0 \mathrm{M} \text { ethanol }\end{array}$ & 3.63 & 6.54 & 58 \\
\hline PdAu NWs & $\begin{array}{l}\text { 1.0 M KOH + } \\
1.0 \mathrm{M} \text { ethanol }\end{array}$ & 10.8 & - & 59 \\
\hline 2D PdPtCu NRs & $\begin{array}{l}1.0 \mathrm{M} \mathrm{KOH} \mathrm{+} \\
1.0 \mathrm{M} \text { ethanol }\end{array}$ & 12.42 & 13.25 & 60 \\
\hline 2D PdAgPt & $\begin{array}{l}1.0 \mathrm{M} \mathrm{KOH} \mathrm{+} \\
1.0 \mathrm{M} \text { ethanol }\end{array}$ & 3.33 & & 61 \\
\hline Au@PtIr/C & $\begin{array}{l}1.0 \mathrm{M} \mathrm{KOH} \mathrm{+} \\
1.0 \mathrm{M} \text { ethanol }\end{array}$ & 58 & - & 62 \\
\hline $\mathbf{P t}_{3} \mathrm{Ag}$ & $\begin{array}{l}1.0 \mathrm{M} \mathrm{KOH} \mathrm{+} \\
1.0 \mathrm{M} \text { ethanol }\end{array}$ & 6.1 & 28 & 63 \\
\hline SANi-PtNWs & $\begin{array}{l}1.0 \mathrm{M} \mathrm{KOH} \mathrm{+} \\
1.0 \mathrm{M} \text { ethanol }\end{array}$ & 5.60 & - & 64 \\
\hline PtRhCu CNBs & $\begin{array}{l}1.0 \mathrm{M} \mathrm{KOH} \mathrm{+} \\
1.0 \mathrm{M} \text { ethanol }\end{array}$ & 4.09 & 14.9 & 65 \\
\hline $\mathrm{Au} @ \mathbf{P t} / \mathrm{C}$ & $\begin{array}{l}1.0 \mathrm{M} \mathrm{KOH} \mathrm{+} \\
1.0 \mathrm{M} \text { ethanol }\end{array}$ & 6.9 & - & 62 \\
\hline PtRhNi-ANAs & $\begin{array}{l}1.0 \mathrm{M} \mathrm{KOH} \mathrm{+} \\
1.0 \mathrm{M} \text { ethanol }\end{array}$ & 1.39 & 7.97 & 66 \\
\hline
\end{tabular}

Notes: The mass activities were calculated by normalizing the activity to the mass of Pd (for Pd-based catalysts), Pt (for Pt-based catalysts), or Pt + Pd (for Pt-Pd based catalysts), unless otherwise stated. The highly strained ultrathin Au-Ag-Pd alloy nanowires obtained in this work demonstrate a mass activity of 13.6 $\mathrm{A} \mathrm{mgPd}^{-1}$ in $1 \mathrm{M} \mathrm{KOH}+1 \mathrm{M}$ ethanol, which is a record-high value among all activities of Pdbased catalysts reported in the literature under the similar conditions. 


\section{References:}

1. Zhang, S.; Zhang, L.; Liu, Z.; Liu, M.; Fan, Q.; Liu, K.; Gao, C. Robust Synthesis of Ultrathin Au-Ag Nanowires as a High-Surface-Area, Synergistic Substrate for Constructing Efficient Pt-Based Catalysts. J. Mater. Chem. A 2018, 6, 22161-22169.

2. Wu, T.; Fan, J. C.; Li, Q. X.; Shi, P. H.; Xu, Q. J.; Min, Y. L. Palladium Nanoparticles Anchored on Anatase Titanium Dioxide-Black Phosphorus Hybrids with Heterointerfaces: Highly Electroactive and Durable Catalysts for Ethanol Electrooxidation. Adv. Energy Mater. 2018, 8, 1701799.

3. Chierchie, T.; Mayer, C.; Lorenz, W. J. Structural Changes of Surface Oxide Layers on Palladium. J. Electroanal. Chem. Interfacial Electrochem. 1982, 135, 211-220.

4. Zhang, L.; Sha, X.; Fan, Q.; Han, L.; Yin, Y.; Gao, C. Gold Nanoshurikens with Uniform Sharp Tips for Chemical Sensing by the Localized Surface Plasmon Resonance. Nanoscale 2017, 9, 17037-17043.

5. Butler, J. N. Solubility and Complex Formation Equilibria of Silver Chloride in Anhydrous Dimethylformamide. J. Phys. Chem. 1968, 72, 3288-3292.

6. Zhang, S.; Zhang, L.; Liu, K.; Liu, M.; Yin, Y.; Gao, C. Digestive Ripening in the Formation of Monodisperse Silver Nanospheres. Mater. Chem. Front. 2018, 2, 1328-1333.

7. Chung, D. Y.; Lee, K.-J.; Sung, Y. E. Methanol Electro-Oxidation on the Pt Surface: Revisiting the Cyclic Voltammetry Interpretation. J. Phys. Chem. C 2016, 120, 9028-9035.

8. Cai, K.; Liao, Y.; Zhang, H.; Liu, J.; Lu, Z.; Huang, Z.; Chen, S.; Han, H. Controlled Synthesis of AuIsland-Covered Pd Nanotubes with Abundant Heterojunction Interfaces for Enhanced Electrooxidation of Alcohol. ACS Appl. Mater. Interfaces 2016, 8, 12792-12797.

9. Li, S.; Lai, J.; Luque, R.; Xu, G. Designed Multimetallic Pd Nanosponges with Enhanced Electrocatalytic Activity for Ethylene Glycol and Glycerol Oxidation. Energy Environ. Sci. 2016, 9, 30973102. 
10. Gao, F.; Zhang, Y.; Ren, F.; Shiraishi, Y.; Du, Y. Universal Surfactant-Free Strategy for Self-Standing 3D Tremella-Like Pd-M ( $\mathrm{M}=\mathrm{Ag}, \mathrm{Pb}$, and $\mathrm{Au})$ Nanosheets for Superior Alcohols Electrocatalysis. $A d v$. Funct. Mater. 2020, 30, 2000255.

11. Zhao, M.; Lyu, Z.; Xie, M.; Hood, Z. D.; Cao, Z.; Chi, M.; Xia, Y. Pd-Ru Alloy Nanocages with a Face-Centered Cubic Structure and Their Enhanced Activity toward the Oxidation of Ethylene Glycol and Glycerol. Small Methods 2020, 4, 1900843.

12. Xu, H.; Wang, J.; Yan, B.; Li, S.; Wang, C.; Shiraishi, Y.; Yang, P.; Du, Y. Facile Construction of Fascinating Trimetallic PdAuAg Nanocages with Exceptional Ethylene Glycol and Glycerol Oxidation Activity. Nanoscale 2017, 9, 17004-17012.

13. Karuppasamy, L.; Chen, C. Y.; Anandan, S.; Wu, J. J. Low-and High-Index Faceted Pd Nanocrystals Embedded in Various Oxygen-Deficient $\mathrm{WO}_{\mathrm{x}}$ Nanostructures for Electrocatalytic Oxidation of Alcohol (EOA) and Carbon Monoxide (CO). ACS Appl. Mater. Interfaces 2019, 11, 10028-10041.

14. Xu, H.; Yan, B.; Zhang, K.; Wang, J.; Li, S.; Wang, C.; Shiraishi, Y.; Du, Y.; Yang, P. UltrasonicAssisted Synthesis of N-Doped Graphene-Supported Binary PdAu Nanoflowers for Enhanced ElectroOxidation of Ethylene Glycol and Glycerol. Electrochim. Acta 2017, 245, 227-236.

15. Xing, X. L.; Zhao, Y. F.; Li, H.; Wang, C. T.; Li, Q. X.; Cai, W. B. High Performance Ag Rich PdAg Bimetallic Electrocatalyst for Ethylene Glycol Oxidation in Alkaline Media. J. Electrochem. Soc. 2018, $165, \mathrm{~J} 3259-\mathrm{J} 3265$.

16. Wang, C.; Song, P. P.; Gao, F.; Song, T. X.; Zhang, Y. P.; Chen, C. Y.; Li, L.; Jin, L. J.; Du, Y. K. Precise Synthesis of Monodisperse PdAg Nanoparticles for Size-Dependent Electrocatalytic Oxidation Reactions. J. Colloid Interface Sci. 2019, 544, 284-292.

17. Guo, X. Z.; Shang, H. Y.; Guo, J.; Xu, H.; Du, Y. K. Ultrafine Two-Dimensional Alloyed PdCu Nanosheets-Constructed Three-Dimensional Nanoflowers Enable Efficient Ethylene Glycol Electrooxidation. Appl. Surf. Sci. 2019, 481, 1532-1537. 
18. Xu, H.; Song, P.; Fernandez, C.; Wang, J.; Zhu, M.; Shiraishi, Y.; Du, Y. Sophisticated Construction of Binary $\mathrm{PdPb}$ Alloy Nanocubes as Robust Electrocatalysts toward Ethylene Glycol and Glycerol Oxidation. ACS Appl. Mater. Interfaces 2018, 10, 12659-12665.

19. Cui, X. X.; Li, Y. L.; Zhao, M. Y.; Xu, Y. S.; Chen, L. L.; Yang, S. G.; Wang, Y. Facile Growth of Ultra-Small Pd Nanoparticles on Zeolite-Templated Mesocellular Graphene Foam for Enhanced Alcohol Electrooxidation. Nano Res. 2019, 12, 351-356.

20. Jin, L. J.; Xu, H.; Chen, C. Y.; Song, T. X.; Wang, C.; Wang, Y.; Shang, H. Y.; Du, Y. K. Uniform $\mathrm{PdCu}$ Coated Te Nanowires as Efficient Catalysts for Electrooxidation of Ethylene Glycol. J. Colloid Interface Sci. 2019, 540, 265-271.

21. Xiong, Z.; Xu, H.; Li, S.; Gu, Z.; Yan, B.; Guo, J.; Du, Y. Concave Pd-Ru Nanocubes Bounded with High Active Area for Boosting Ethylene Glycol Electrooxidation. Appl. Surf. Sci. 2018, 427, 83-89.

22. Li, W.; Huang, Y.; Tang, D.; Zhang, T.; Wang, Y. A New Composite Support for Pd Catalysts for Ethylene Glycol Electrooxidation in Alkaline Solution: Effect of $(\mathrm{Ru}, \mathrm{Sn}) \mathrm{O}_{2}$ Solid Solution. Electrochim. Acta 2015, 174, 178-184.

23. Lai, J.; Lin, F.; Tang, Y.; Zhou, P.; Chao, Y.; Zhang, Y.; Guo, S. Efficient Bifunctional Polyalcohol Oxidation and Oxygen Reduction Electrocatalysts Enabled by Ultrathin PtPdM ( $\mathrm{M}=\mathrm{Ni}, \mathrm{Fe}, \mathrm{Co})$ Nanosheets. Adv. Energy Mater. 2019, 9, 1800684.

24. Hong, W.; Shang, C.; Wang, J.; Wang, E. Bimetallic PdPt Nanowire Networks with Enhanced Electrocatalytic Activity for Ethylene Glycol and Glycerol Oxidation. Energy Environ. Sci. 2015, 8, 29102915.

25. Zheng, J.-N.; He, L.-L.; Chen, F.-Y.; Wang, A.-J.; Xue, M.-W.; Feng, J.-J. A Facile General Strategy for Synthesis of Palladium-Based Bimetallic Alloyed Nanodendrites with Enhanced Electrocatalytic Performance for Methanol and Ethylene Glycol Oxidation. J. Mater. Chem. A 2014, 2, 12899-12906.

26. Gao, J.; Mao, M.; Li, P.; Liu, R.; Song, H.; Sun, K.; Zhang, S. Segmentation and Re-Encapsulation of Porous PtCu Nanoparticles by Generated Carbon Shell for Enhanced Ethylene Glycol Oxidation and Oxygen-Reduction Reaction. ACS Appl. Mater. Interfaces 2020, 12, 6298-6308. 
27. Feng, Y.; Bu, L.; Guo, S.; Guo, J.; Huang, X. 3D Platinum-Lead Nanowire Networks as Highly Efficient Ethylene Glycol Oxidation Electrocatalysts. Small 2016, 12, 4464-4470.

28. Du, H. Y.; Wang, K.; Tsiakaras, P.; Shen, P. K. Excavated and Dendritic Pt-Co Nanocubes as Efficient Ethylene Glycol and Glycerol Oxidation Electrocatalysts. Appl. Catal., B 2019, 258, 7.

29. Shao, F.-Q.; Lin, X.-X.; Feng, J.-J.; Yuan, J.; Chen, J.-R.; Wang, A.-J. Simple Fabrication of CoreShell AuPt@Pt Nanocrystals Supported on Reduced Graphene Oxide for Ethylene Glycol Oxidation and Hydrogen Evolution Reactions. Electrochim. Acta 2016, 219, 321-329.

30. Hong, W.; Shang, C.; Wang, J.; Wang, E. Trimetallic PtCuCo Hollow Nanospheres with a Dendritic Shell for Enhanced Electrocatalytic Activity toward Ethylene Glycol Electrooxidation. Nanoscale 2015, 7, 9985-9989.

31. Gao, F.; Zhang, Y.; Song, P.; Wang, J.; Song, T.; Wang, C.; Song, L.; Shiraishi, Y.; Du, Y. PrecursorMediated Size Tuning of Monodisperse PtRh Nanocubes as Efficient Electrocatalysts for Ethylene Glycol Oxidation. J. Mater. Chem. A 2019, 7, 7891-7896.

32. Li, Z. Y.; Zhou, J.; Tang, L. S.; Fu, X. P.; Wei, H.; Xue, M.; Zhao, Y. L.; Jia, C. J.; Li, X. M.; Chu, H. B.; Li, Y. Hydroxyl-Rich Ceria Hydrate Nanoparticles Enhancing the Alcohol Electrooxidation Performance of Pt Catalysts. J. Mater. Chem. A 2018, 6, 2318-2326.

33. Qi, J.; Benipal, N.; Liang, C.; Li, W. PdAg/CNT Catalyzed Alcohol Oxidation Reaction for HighPerformance Anion Exchange Membrane Direct Alcohol Fuel Cell (Alcohol = Methanol, Ethanol, Ethylene Glycol and Glycerol). Appl. Catal., B 2016, 199, 494-503.

34. Zalineeva, A.; Serov, A.; Padilla, M.; Martinez, U.; Artyushkova, K.; Baranton, S.; Coutanceau, C.; Atanassov, P. B. Self-Supported $\mathrm{Pd}_{\mathrm{x}} \mathrm{Bi}$ Catalysts for the Electrooxidation of Glycerol in Alkaline Media. J. Am. Chem. Soc. 2014, 136, 3937-3945.

35. Liu, M. T.; Chen, L. X.; Li, D. N.; Wang, A. J.; Zhang, Q. L.; Feng, J. J. One-Pot Controlled Synthesis of AuPd@Pd Core-Shell Nanocrystals with Enhanced Electrocatalytic Performances for Formic Acid Oxidation and Glycerol Oxidation. J. Colloid Interface Sci. 2017, 508, 551-558. 
36. Song, P.; Feng, J.-J.; Guo, F.-Y.; Wang, A.-J. Simple Polyol Synthesis of Porous Coral-Like Palladium-Silver Alloy Nanostructures with Enhanced Electrocatalytic Activity for Glycerol Oxidation Reaction. J. Mater. Chem. A 2015, 3, 15920-15926.

37. Shang, H.; Xu, H.; Wang, C.; Jin, L.; Chen, C.; Zhou, G.; Wang, Y.; Du, Y. General Synthesis of Pd$\mathrm{Pm}(\mathrm{Pm}=\mathrm{Ga}$, in, Sn, Pb, Bi) Alloy Nanosheet Assemblies for Advanced Electrocatalysis. Nanoscale 2020, $12,3411-3417$.

38. Yang, F.; Ye, J.; Yuan, Q.; Yang, X.; Xie, Z.; Zhao, F.; Zhou, Z.; Gu, L.; Wang, X. Ultrasmall Pd$\mathrm{Cu}-\mathrm{Pt}$ Trimetallic Twin Icosahedrons Boost the Electrocatalytic Performance of Glycerol Oxidation at the Operating Temperature of Fuel Cells. Adv. Funct. Mater. 2020, 30, 1908235.

39. Hong, W.; Wang, J.; Wang, E. Scalable Synthesis of Cu-Based Ultrathin Nanowire Networks and Their Electrocatalytic Properties. Nanoscale 2016, 8, 4927-4932.

40. Kim, Y.; Kim, H.; Kim, W. B. PtAg Nanotubes for Electrooxidation of Ethylene Glycol and Glycerol in Alkaline Media. Electrochem. Commun. 2014, 46, 36-39.

41. Yang, Z. Z.; Lin, X. X.; Zhang, X. F.; Wang, A. J.; Zhu, X. Y.; Feng, J. J. Bimetallic PtPd Alloyed Core-Shell Nanodendrites Supported on Reduced Graphene Oxide: One-Pot Green Synthesis and Efficient Electrocatalytic Performances for Glycerol Oxidation and Hydrogen Evolution. J. Alloys Compd. 2018, $735,2123-2132$.

42. Wang, A. L.; Zhu, L.; Yun, Q.; Han, S.; Zeng, L.; Cao, W.; Meng, X.; Xia, J.; Lu, Q. Bromide Ions Triggered Synthesis of Noble Metal-Based Intermetallic Nanocrystals. Small 2020, e2003782.

43. Yin, P. F.; Zhou, M.; Chen, J.; Tan, C.; Liu, G.; Ma, Q.; Yun, Q.; Zhang, X.; Cheng, H.; Lu, Q.; Chen, B.; Chen, Y.; Zhang, Z.; Huang, J.; Hu, D.; Wang, J.; Liu, Q.; Luo, Z.; Liu, Z.; Ge, Y.; Wu, X. J.; Du, X. W.; Zhang, H. Synthesis of Palladium-Based Crystalline@Amorphous Core-Shell Nanoplates for Highly Efficient Ethanol Oxidation. Adv. Mater. 2020, 32, e2000482.

44. Wang, M.; Ding, R.; Xiao, Y.; Wang, H.; Wang, L.; Chen, C. M.; Mu, Y.; Wu, G. P.; Lv, B. CoP/rGOPd Hybrids with Heterointerfaces as Highly Active Catalysts for Ethanol Electrooxidation. ACS Appl. Mater. Interfaces 2020, 12, 28903-28914. 
45. Lv, H.; Sun, L.; Chen, X.; Xu, D.; Liu, B. One-Step Fabrication of Trimetallic Core-Shell Au@PdAuCu Mesoporous Nanospheres for Ethanol Electrooxidation. Green Chem. 2019, 21, 2043-2051. 46. Lv, H.; Wang, Y.; Lopes, A.; Xu, D.; Liu, B. Ultrathin PdAg Single-Crystalline Nanowires Enhance Ethanol Oxidation Electrocatalysis. Appl. Catal., B 2019, 249, 116-125.

47. Lv, H.; Sun, L.; Zou, L.; Xu, D.; Yao, H.; Liu, B. Size-Dependent Synthesis and Catalytic Activities of Trimetallic PdAgCu Mesoporous Nanospheres in Ethanol Electrooxidation. Chem. Sci. 2019, 10, 19861993.

48. Wu, T.; Ma, Y.; Qu, Z.; Fan, J.; Li, Q.; Shi, P.; Xu, Q.; Min, Y. Black Phosphorus-Graphene Heterostructure-Supported Pd Nanoparticles with Superior Activity and Stability for Ethanol ElectroOxidation. ACS Appl. Mater. Interfaces 2019, 11, 5136-5145.

49. Yuan, X.; Zhang, Y.; Cao, M.; Zhou, T.; Jiang, X.; Chen, J.; Lyu, F.; Xu, Y.; Luo, J.; Zhang, Q.; Yin, Y. $\mathrm{Bi}(\mathrm{OH})_{3} / \mathrm{PdBi}$ Composite Nanochains as Highly Active and Durable Electrocatalysts for Ethanol Oxidation. Nano Lett. 2019, 19, 4752-4759.

50. Huang, W.; Kang, X.; Xu, C.; Zhou, J.; Deng, J.; Li, Y.; Cheng, S. 2D PdAg Alloy Nanodendrites for Enhanced Ethanol Electroxidation. Adv. Mater. 2018, 30, 1706962.

51. Yang, H.; Yu, Z.; Li, S.; Zhang, Q.; Jin, J.; Ma, J. Ultrafine Palladium-Gold-Phosphorus Ternary Alloyed Nanoparticles Anchored on Ionic Liquids-Noncovalently Functionalized Carbon Nanotubes with Excellent Electrocatalytic Property for Ethanol Oxidation Reaction in Alkaline Media. J. Catal. 2017, 353, 256-264.

52. Chen, Y.; Fan, Z.; Luo, Z.; Liu, X.; Lai, Z.; Li, B.; Zong, Y.; Gu, L.; Zhang, H. High-Yield Synthesis of Crystal-Phase-Heterostructured 4H/fcc Au@Pd Core-Shell Nanorods for Electrocatalytic Ethanol Oxidation. Adv. Mater. 2017, 29, 1701331.

53. Karuppasamy, L.; Anandan, S.; Chen, C.-Y.; Wu, J. J. Sonochemical Synthesis of PdAg/rGO Nanocomposite as an Efficient Electrocatalyst for Both Ethanol Oxidation and Oxygen Reduction Reaction with High CO Tolerance. Electrocatalysis 2017, 8, 430-441. 
54. Huang, W.; Ma, X. Y.; Wang, H.; Feng, R.; Zhou, J.; Duchesne, P. N.; Zhang, P.; Chen, F.; Han, N.; Zhao, F.; Zhou, J.; Cai, W. B.; Li, Y. Promoting Effect of Ni(OH) $)_{2}$ on Palladium Nanocrystals Leads to Greatly Improved Operation Durability for Electrocatalytic Ethanol Oxidation in Alkaline Solution. Adv. Mater. 2017, 29, 1703057.

55. Chen, L.; Lu, L.; Zhu, H.; Chen, Y.; Huang, Y.; Li, Y.; Wang, L. Improved Ethanol Electrooxidation Performance by Shortening Pd-Ni Active Site Distance in Pd-Ni-P Nanocatalysts. Nat. Commun. 2017, 8, 14136.

56. Chen, Z.; Zhang, J.; Zhang, Y.; Liu, Y.; Han, X.; Zhong, C.; Hu, W.; Deng, Y. NiO-Induced Synthesis of PdNi Bimetallic Hollow Nanocrystals with Enhanced Electrocatalytic Activities toward Ethanol and Formic Acid Oxidation. Nano Energy 2017, 42, 353-362.

57. Zhu, C.; Shi, Q.; Fu, S.; Song, J.; Xia, H.; Du, D.; Lin, Y. Efficient Synthesis of MCu (M = Pd, Pt, and $\mathrm{Au}$ ) Aerogels with Accelerated Gelation Kinetics and Their High Electrocatalytic Activity. Adv. Mater. 2016, 28, 8779-8783.

58. Cai, B.; Wen, D.; Liu, W.; Herrmann, A. K.; Benad, A.; Eychmuller, A. Function-Led Design of Aerogels: Self-Assembly of Alloyed PdNi Hollow Nanospheres for Efficient Electrocatalysis. Angew. Chem. Int. Ed. 2015, 54, 13101-13105.

59. Hong, W.; Wang, J.; Wang, E. Facile Synthesis of Highly Active PdAu Nanowire Networks as SelfSupported Electrocatalyst for Ethanol Electrooxidation. ACS Appl. Mater. Interfaces 2014, 6, 9481-9487.

60. Wang, W.; Zhang, X.; Zhang, Y.; Chen, X.; Ye, J.; Chen, J.; Lyu, Z.; Chen, X.; Kuang, Q.; Xie, S.; Xie, Z. Edge Enrichment of Ultrathin 2D PdPtCu Trimetallic Nanostructures Effectuates Top-Ranked Ethanol Electrooxidation. Nano Lett. 2020, 20, 5458-5464.

61. Lu, W.; Xia, X.; Wei, X.; Li, M.; Zeng, M.; Guo, J.; Cheng, S. Nanoengineering 2D Dendritic PdAgPt Nanoalloys with Edge-Enriched Active Sites for Enhanced Alcohol Electroxidation and Electrocatalytic Hydrogen Evolution. ACS Appl. Mater. Interfaces 2020, 12, 21569-21578. 
62. Liang, Z.; Song, L.; Deng, S.; Zhu, Y.; Stavitski, E.; Adzic, R. R.; Chen, J.; Wang, J. X. Direct 12Electron Oxidation of Ethanol on a Ternary Au(Core)-PtIr(Shell) Electrocatalyst. J. Am. Chem. Soc. 2019, 141, 9629-9636.

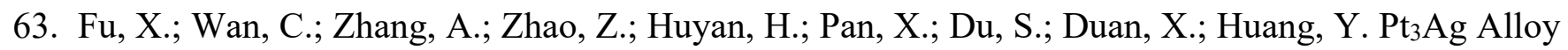
Wavy Nanowires as Highly Effective Electrocatalysts for Ethanol Oxidation Reaction. Nano Res. 2020, $13,1472-1478$.

64. Li, M.; Duanmu, K.; Wan, C.; Cheng, T.; Zhang, L.; Dai, S.; Chen, W.; Zhao, Z.; Li, P.; Fei, H.; Zhu, Y.; Yu, R.; Luo, J.; Zang, K.; Lin, Z.; Ding, M.; Huang, J.; Sun, H.; Guo, J.; Pan, X.; Goddard, W. A.; Sautet, P.; Huang, Y.; Duan, X. Single-Atom Tailoring of Platinum Nanocatalysts for High-Performance Multifunctional Electrocatalysis. Nat. Catal. 2019, 2, 495-503.

65. Han, S.-H.; Liu, H.-M.; Chen, P.; Jiang, J.-X.; Chen, Y. Porous Trimetallic PtRhCu Cubic Nanoboxes for Ethanol Electrooxidation. Adv. Energy Mater. 2018, 8, 1801326.

66. Liu, H.; Li, J.; Wang, L.; Tang, Y.; Xia, B. Y.; Chen, Y. Trimetallic PtRhNi Alloy Nanoassemblies as Highly Active Electrocatalyst for Ethanol Electrooxidation. Nano Res. 2017, 10, 3324-3332. 